\title{
J. BOUZITAT
}

\section{Sur la méthode de Wolfe et la méthode de Dantzig en programmation quadratique convexe}

Revue française d'automatique, d'informatique et de recherche opérationnelle. Recherche opérationnelle, tome 13, n 2 (1979), p. 151-184.

<http://www.numdam.org/item?id=RO_1979_13_2_151_0>

(C) AFCET, 1979, tous droits réservés.

L'accès aux archives de la revue « Revue française d'automatique, d'informatique et de recherche opérationnelle. Recherche opérationnelle » implique l'accord avec les conditions générales d'utilisation (http://www.numdam.org/ legal.php). Toute utilisation commerciale ou impression systématique est constitutive d'une infraction pénale. Toute copie ou impression de ce fichier doit contenir la présente mention de copyright.

\section{Numdam}

Article numérisé dans le cadre du programme

Numérisation de documents anciens mathématiques

http://www.numdam.org/ 


\title{
SUR LA MÉTHODE DE WOLFE ET LA MÉTHODE DE DANTZIG EN PROGRAMMATION QUADRATIQUE CONVEXE $\left({ }^{*}\right)$
}

\author{
par J. Bouzitat $\left({ }^{1}\right)$
}

Résumé. - La méthode de Wolfe et la méthode de Dantzig résolvent les problèmes de programmation quadratique convexe à contraintes linéaires par des algorithmes apparentés à celui du Simplexe. Elles s'appuient sur les conditions de Kuhn et Tucker, qui sont alors nécessaires et suffisantes. Le présent article expose ces méthodes et en donne des justifications théoriques complètes qui, à la connaissance de l'auteur, sont en grande partie originales.

La méthode de Wolfe est ici complétée et se révèle alors plus efficace que ne le laissaient croire des phénomènes de " blocage " apparent, dont l'étude montre qu'ils ne compromettent pas la convergence du processus complété. La " forme courte » de la méthode suffit ainsi pour résoudre tous les problèmes non paramétriques, et la "forme longue " peut être réservée aux problèmes paramétriques.

Lajustification présentée pour la méthode de Dantzig ne s'appuie pas sur l'étude directe de la suite des tableaux de calcul, mais sur la convexité de la fonction quadratique à minimiser. Elle s'en trouve considérablement allégée.

Enfin la présentation générale des méthodes de Wolfe et de Dantzig est illustrée par un exemple numérique complètement traité, qui permet de les comparer.

Abstract. - Both Wolfe's and Dantzig's methods solve linear-constrained convex quadratic programming problems by simplex-like algorithms. They use the Kuhn-Tucker conditions, which are necessary and sufficient for such problems. This paper presents those two methods, with complete theoretical proofs the greater part of which, to the author's knowledge, is new.

Wolfe's method receives here a complement and is then found to be more efficient than it previously appeared, on account of "blocking" phenomena which are proved not to stop the convergent process of the completed algorithm. The "short form" of the method is consequently applicable to solve any nonparametric problem, and the "long form" may be reserved for parametric problems only.

The present proof of Dantzig's algorithm convergence is not based on the direct study of computation schemata, but uses the convexity of the quadratic function to be minimized, which leads to a quite simple proof.

The general presentation of Wolfe's and Dantzig's methods is illustrated by a numerical problem which is solved by both of them, so as to permit a comparison.

(*) Reçu mars 1978.

(1) Université de Paris-I - Panthéon-Sorborne.

R.A.I.R.O. Recherche opérationnelle/Operations Research, 0399-0842/1979/151/\$ 4.00

(c) Bordas-Dunod 


\section{PROBLÈME DE PROGRAMMATION QUADRATIQUE CONVEXE A CONTRAINTES} LINEAIRES

On considère le problème paramétrique suivant :

$$
\text { Minimiser } f(\lambda, x)=\lambda \underset{(1, n)}{p^{\prime}} x+\frac{1}{2} x^{\prime} \underset{(n, n)}{C} x \text {, }
$$

sous les contraintes $\left({ }^{2}\right)$ :

$$
\underset{(m, n)}{A} x=\underset{(m, 1)}{b} \quad \text { et } \quad x \geqq \underset{(n, 1)}{0},
$$

$x \in \mathbf{R}^{n}$ étant un vecteur inconnu, $\lambda$ étant un paramètre réel positif ou nul, $(n, 1)$

$C$ étant une matrice symétrique réelle telle que $x^{\prime} \quad C \quad x \geqq 0, \forall x \in \mathbf{R}^{n}$. $(n, n)$

La forme quadratique $x^{\prime} C x$ est alors dite semi-définie positive, et elle définit une fonction convexe.

\subsection{Propriétés générales}

1)

$$
\left[x^{\prime} C x=0\right] \Rightarrow[C x=0] \text {. }
$$

En effet

$$
\left[x^{\prime} C x=0\right] \Rightarrow\left[(y+t x)^{\prime} C(y+t x)=y^{\prime} C y+2 t y^{\prime} C x\right]
$$

et

$y^{\prime} C y+2 t y^{\prime} C x \geqq 0, \forall t \in \mathbf{R}$ et $\left.\forall y \in \mathbf{R}^{n}\right]$

$$
\Rightarrow \quad\left[y^{\prime} C x=0, \forall y \in \mathbf{R}^{n}\right] \Rightarrow[C x=0] .
$$

2) Si $\bar{x}$ est solution du problème posé, l'ensemble des solutions est

$$
\left\{x=\bar{x}+z \mid \lambda p^{\prime} z=0, C z=0, A z=0, \bar{x}+z \geqq 0\right\} .
$$

(Cet ensemble peut d'ailleurs se réduire à $\{\bar{x}\}$. C'est le cas, en particulier, si $\left[x^{\prime} C x=0\right] \Rightarrow[x=0]$.)

En effet tout point $x$ de cet ensemble est solution du problème.

Réciproquement, si $\bar{x}$ et $\bar{x}+z$ sont solutions du problème, il en est de même de $\bar{x}+t z, \forall t \in[0,1]$ puisque la fonction $f$ est convexe en $x$, de sorte que

a) $\lambda p^{\prime}(\bar{x}+t z)+\frac{1}{2}(\bar{x}+t z)^{\prime} C(\bar{x}+t z)=\lambda p^{\prime} \bar{x}+\bar{x}^{\prime} C \bar{x}, \quad \forall t \in[0,1]$,

$$
\left(\lambda p^{\prime} z+\bar{x}^{\prime} C z\right) t+\frac{1}{2}\left(z^{\prime} C z\right) t^{2}=0, \quad \forall t \in[0,1] \text {, }
$$

$\left({ }^{2}\right)$ Tous les types de contraintes linéaires peuvent être mis sous cette forme. On pourrait aussi se donner les contraintes sous la forme $A x \leqq b, x \geqq 0(c f . \S 4.1)$, ou sous d'autres formes plus générales. 
d'où

b)

$$
z^{\prime} C z=0 \quad \text { et } \quad C z=0 \quad \text { (d'après } 1 \text { ), } \quad \lambda p^{\prime} z=0 \text {, }
$$

$A(\bar{x}+t z)=A \bar{x}, \quad \forall t \in[0,1]$ et $A z=0$, $\bar{x}+t z \geqq 0, \quad \forall t \in[0,1] \quad$ et $\quad \bar{x}+z \geqq 0$.

$$
F(\lambda)=\min _{x}\{f(\lambda, x) \mid A x=b \text { et } x \geqq 0\}=f\left(\lambda, x_{\lambda}\right)
$$

est une fonction concave de $\lambda$, et $p^{\prime} x_{\lambda}$ est une fonction non croissante de $\lambda$.

En effet

$$
\begin{aligned}
& F\left(t \lambda_{1}+(1-t) \lambda_{2}\right)=f\left(t \lambda_{1}+(1-t) \lambda_{2}, x_{t \lambda_{1}+(1-t) \lambda_{2}}\right)=f\left(\lambda, x_{\lambda}\right) \\
&=t f\left(\lambda_{1}, x_{\lambda}\right)+(1-t) f\left(\lambda_{2}, x_{\lambda}\right) \geqq t F\left(\lambda_{1}\right)+(1-t) F\left(\lambda_{2}\right), \quad \forall t \in[0,1] .
\end{aligned}
$$

D'autre part

$$
f\left(\lambda, x_{\lambda}\right)+f\left(\mu, x_{\mu}\right) \leqq f\left(\lambda, x_{\mu}\right)+f\left(\mu, x_{\lambda}\right),
$$

d'où

$$
\lambda p^{\prime} x_{\lambda}+\mu p^{\prime} x_{\mu} \leqq \lambda p^{\prime} x_{\mu}+\mu p^{\prime} x_{\lambda}
$$

ou

$$
(\lambda-\mu)\left(p^{\prime} x_{-\lambda}-p^{\prime} x_{\mu}\right) \leqq 0 .
$$

Remarque. - Puisque $f(\lambda, x) \geqq f\left(\lambda, x_{\lambda}\right), \forall x \mid A x=b$ et $x \geqq 0$,

$$
\left[A x=b, x \geqq 0, p^{\prime} x \leqq p^{\prime} x_{\lambda}\right] \Rightarrow\left[x^{\prime} C x \geqq x_{\lambda}^{\prime} C x_{\lambda}\right],
$$

de sorte que

$$
x_{\lambda}^{\prime} C x_{\lambda}=\min \left\{x^{\prime} C x \mid A x=b, x \geqq 0, p^{\prime} x \leqq p^{\prime} x_{\lambda}\right\} .
$$

\subsection{Effet de perturbations portant sur la matrice $C$}

Si la forme $x^{\prime} C x$ est semi-définie positive, et non définie positive, il se peut que la solution du problème posé existe sans être unique. Soit alors $\hat{x}$ la solution de norme $\left(\hat{x}^{\prime} \hat{x}\right)^{1 / 2}$ minimale. Cette solution existe et est unique puisque l'ensemble des solutions est fermé et convexe.

Considérons le problème déduit du problème posé par perturbation de la matrice $C$, remplacée par $(C+\varepsilon I)$, où $I$ est une matrice unité et où $\varepsilon$ est un scalaire positif que l'on fera tendre vers zéro.

vol. $13, \mathrm{n}^{\circ} 2$, mai 1979 
La forme $x^{\prime}(C+\varepsilon I) x$ étant définie positive, ce problème a une solution unique $\hat{x}_{\varepsilon}$, minimisant la fonction économique

$$
f_{\varepsilon}(\lambda, x)=\lambda p^{\prime} x+\frac{1}{2} x^{\prime}(C+\varepsilon I) x=f(\lambda, x)+\frac{\varepsilon}{2} x^{\prime} x
$$

sous les contraintes $A x=b$ et $x \geqq 0$.

D'après la définition de $\hat{x}$ et $\hat{x}_{\varepsilon}$,

$$
\begin{aligned}
& f(\lambda, \hat{x})+\frac{\varepsilon}{2} \hat{x}^{\prime} \hat{x}=f_{\varepsilon}(\lambda, \hat{x}) \geqq f_{\varepsilon}\left(\lambda, \hat{x}_{\varepsilon}\right)=f\left(\lambda, \hat{x}_{\varepsilon}\right)+\frac{\varepsilon}{2} \hat{x}_{\varepsilon}^{\prime} \hat{x}_{\varepsilon} \geqq f(\lambda, \hat{x})+\frac{\varepsilon}{2} \hat{x}_{\varepsilon}^{\prime} \hat{x}_{\varepsilon} . \\
& \text { D'où } \quad \lim _{\varepsilon \rightarrow 0} f_{\varepsilon}\left(\lambda, \hat{x}_{\varepsilon}\right)=f(\lambda, \hat{x}) \text { et } \hat{x}_{\varepsilon}^{\prime} \hat{x}_{\varepsilon} \leqq \hat{x}^{\prime} \hat{x}
\end{aligned}
$$

(On montre de même que $\hat{x}_{\varepsilon}^{\prime} \hat{x}_{\varepsilon}$ est fonction non croissante de $\varepsilon>0$.)

Il en résulte que

$$
\lim _{\varepsilon \rightarrow 0} \hat{x}_{\varepsilon}=\hat{x}
$$

En effet, s'il n'en était pas ainsi, l'ensemble des $\hat{x}_{\varepsilon} \mid \varepsilon>0$ étant borné, il existerait une suite convergente $\hat{x}_{\varepsilon_{1}}, \hat{x}_{\varepsilon_{2}}, \ldots, \hat{x}_{\varepsilon_{n}}, \ldots$ avec $\varepsilon_{1}>\varepsilon_{2}>\ldots>\varepsilon_{n}>\ldots$ et $\varepsilon_{n} \underset{n \rightarrow \infty}{\longrightarrow} 0$, telle que $\lim _{n \rightarrow \infty} \hat{x}_{\varepsilon_{n}}=x_{0} \neq \hat{x}$.

Puisque $f_{\varepsilon}(\lambda, x)$ est fonction continue de $\varepsilon$ et de $x$, on aurait alors

$$
f\left(\lambda, x_{0}\right)=\lim _{n \rightarrow \infty} f_{\varepsilon_{n}}\left(\lambda, \hat{x}_{\varepsilon_{n}}\right)=f(\lambda, \hat{x}), \quad \text { avec } \quad x_{0}^{\prime} x_{0} \leqq \hat{x}^{\prime} \hat{x},
$$

de sorte que $x_{0}$, vérifiant de plus les contraintes, serait une seconde solution de norme minimum du problème posé. Or il n'en existe pas d'autre que $\hat{x}$.

Ainsi, le remplacement de la forme $x^{\prime} C x$ semi-définie positive par $x^{\prime}(C+\varepsilon I) x$ définie positive $(\varepsilon>0)$ conduit à une solution $\hat{x}_{\varepsilon}$ du problème perturbé qui tend vers la solution $\hat{x}$ de plus petite norme du problème initial lorsque $\varepsilon$ tend vers zéro.

Cette méthode de perturbations permet, au moins en théorie, d'approcher d'aussi près qu'on le veut la solution de plus petite norme (supposée finie) d'un problème à forme quadratique semi-définie positive, par la résolution d'un problème à forme quadratique définie positive, et par conséquent à solution unique.

Les calculs liés à l'emploi de cette méthode risquent cependant d'être lourds et difficiles à programmer. Mais certaines démonstrations peuvent s'en trouver simplifiées. 


\subsection{Conditions de Kuhn et Tucker}

Associons au problème posé le lagrangien (formé sans tenir compte des conditions de signe $x \geqq 0$ ) :

$$
l(x, u)=\lambda \underset{(1, n)}{p^{\prime}} x+\frac{1}{2} x^{\prime} \underset{(n, n)}{C} x+\underset{(1, m)}{u^{\prime}}(\underset{(m, n)}{A} x-\underset{(m, 1)}{b}),
$$

qui est égal à la fonction économique $f(\lambda, x)$ pour tout programme $x$ réalisable.

Les conditions de Kuhn et Tucker s'écrivent :

$$
\begin{aligned}
& \nabla_{x}^{\prime} l(x, u)=\lambda \underset{(n, 1)}{p}+\underset{(n, n)(n, 1)}{C x}+\underset{(n, m)}{A_{(m, 1)}^{\prime}}=\underset{(n, 1)}{v}(\geqq 0), \\
&-\underset{(m, 1)}{b}+\underset{(m, n)(n, 1)}{A}=0, \\
& \underset{(n, 1)}{x} \geqq 0, \quad \underset{(n, 1)}{v} \geqq 0, \quad v_{j} x_{j}=0, \quad \forall j .
\end{aligned}
$$

Ces conditions sont nécessaires parce que les contraintes du problème posé, étant linéaires, sont qualifiées. Elles sont suffisantes parce que les contraintes sont linéaires et la fonction économique à minimiser est convexe.

Les conditions de Kuhn et Tucker sont ici linéaires par rapport à l'ensemble des $n$ variables $x_{j}$ et des $(m+n)$ multiplicateurs $u_{i}$ et $v_{j}$, si l'on excepte les $n$ relations d'exclusion (ou de complémentarité) $v_{j} x_{j}=0$.

Compte tenu des $(n+m)$ égalités linéaires précédentes,

$$
\begin{gathered}
2 f(\lambda, x)=\lambda p^{\prime} x+\left(\lambda p^{\prime}+x^{\prime} C\right) x=\lambda p^{\prime} x+\left(v^{\prime}-u^{\prime} A\right) x \\
2 f(\lambda, x)=\lambda p^{\prime} x-u^{\prime} b+v^{\prime} x
\end{gathered}
$$

le dernier terme (non linéaire) étant nul quand les relations d'exclusion sont vérifiées.

D'après les relations d'exclusion, dans toute solution des conditions de Kuhn et Tucker il y a au plus $(m+n)$ des $(m+2 n)$ variables scalaires $u_{i}$ (de signes quelconques), $v_{j} \geqq 0, x_{j} \geqq 0$ qui soient non nulles.

Si $(x, u, v)$ et $(x+\delta x, u+\delta u, v+\delta v)$ sont deux solutions du système des $(n+m)$ égalités linéaires précédentes,

$$
\left\{\begin{array}{c}
C \delta x+A^{\prime} \delta u=\delta v \\
A \delta x=0
\end{array}\right.
$$

vol. $13, n^{\circ} 2$, mai 1979 
d'où

$$
\delta v^{\prime} . \delta x=\delta x^{\prime} . C \cdot \delta x \geqq 0 .
$$

Si ces deux solutions vérifient toutes les conditions de Kuhn et Tucker,

$$
v^{\prime} x=0, \quad\left(v^{\prime}+\delta v^{\prime}\right)(x+\delta x)=v^{\prime} \cdot \delta x+\delta v^{\prime} \cdot x+\delta v^{\prime} \cdot \delta x=0,
$$

et

$$
\forall j \in\{1,2, \ldots, n\},\left\{\begin{aligned}
{\left[x_{j}>0\right] } & \Rightarrow\left[v_{j}=0\right] \\
& \Rightarrow\left[\delta v_{j} \geqq 0\right] \Rightarrow\left[v_{j} . \delta x_{j}=0 \text { et } \delta v_{j} \cdot x_{j} \geqq 0\right], \\
{\left[v_{j}>0\right] } & \Rightarrow\left[x_{j}=0\right] \\
& \Rightarrow\left[\delta x_{j} \geqq 0\right] \Rightarrow\left[v_{j} . \delta x_{j} \geqq 0 \text { et } \delta v_{j} \cdot x_{j}=0\right],
\end{aligned}\right.
$$

d'où

$$
\forall j \in\{1,2, \ldots, n\}, \quad v_{j} . \delta x_{j}=0 \quad \text { et } \delta v_{j} . x_{j}=0,
$$

puisque $v^{\prime} . \delta x, \delta v^{\prime} . x, \delta v^{\prime} . \delta x$ sont positifs ou nuls et de somme nulle.

Ainsi, s'il existe une solution des conditions de Kuhn et Tucker pour laquelle $x_{j}>0$ (ou $\left.v_{j}>0\right)$, alors dans toute solution de ces conditions $v_{j}=0\left(\right.$ ou $\left.x_{j}=0\right)$. Cette propriété, classique en programmation linéaire, est ainsi étendue à la programmation quadratique convexe à contraintes linéaires.

Il en résulte que l'ensemble des points $(\bar{x}, \bar{u}, \bar{v})$ solutions des conditions de Kuhn et Tucker peut être défini par un système linéaire obtenu à partir de ces conditions par l'annulation d'une variable déterminée $\left(v_{j}\right.$ ou $\left.x_{j}\right)$ dans chacun des $n$ couples de variables complémentaires.

Cet ensemble est donc un tronçon de l'espace $\mathbf{R}^{m+2 n}$, de sorte qu'il peut être défini à partir de ses points extrêmes, et éventuellement de ses directions d'infinitude extrêmes.

C'est ce qui conduit à chercher des solutions de base (au sens de la méthode du Simplexe) du système des conditions linéaires de Kuhn et Tucker $(m+n$ égalités et $2 n$ conditions de signe portant sur $m+2 n$ variables), en leur imposant de vérifier les $n$ relations d'exclusion $v_{j} x_{j}=0, \forall j$.

Tel est le principe de la résolution des problèmes de programmation quadratique convexe à contraintes linéaires par des méthodes simpliciales. Deux de ces méthodes vont être étudiées : la méthode de Wolfe et la méthode de Dantzig.

\section{MÉTHODE DE WOLFE COMPLETTEE}

\subsection{Principe}

On applique la méthode du Simplexe à un système linéaire déduit des conditions linéaires de Kuhn et Tucker par l'introduction de variables 
artificielles positives ou nulles dont on se propose de minimiser la somme, avec une condition supplémentaire excluant la présence simultanée dans la base de deux variables complémentaires $x_{j}$ et $v_{j}$. Les relations d'exclusion $v_{j} x_{j}=0, \forall j$ sont ainsi constamment vérifiées.

L'optimum du problème de programmation quadratique posé, pour lequel les conditions de Kuhn et Tucker sont nécessaires et suffisantes, est atteint quand toutes les variables artificielles sont annulées. (Dès qu'une variable artificielle s'annule en sortant de la base, elle peut être supprimée.)

Mais, en raison de la restriction imposée par le respect des relations d'exclusion, il n'est pas sûr a priori que la méthode permette toujours d'attejndre l'optimum quand il existe. Et certains exemples ont conduit à penser que le processus pouvait en effet « se bloquer " pour une valeur strictement positive de la somme des variables artificielles, même quand cette somme a un minimum nul. Nous verrons cependant qu'un tel blocage n'est qu'apparent, et que le processus de calcul peut être aménagé de telle manière qu'il conduise toujours à l'optimum quand cet optimum existe, pourvu que l'éventualité d'un « cyclage " soit écartée (cette réserve, d'une faible importance pratique, accompagnant toujours l'application de la méthode du Simplexe).

La forme courte du processus résout le problème de minimisation de $f(\lambda, x)$ pour une valeur déterminée de $\lambda$. La forme longue du processus résout le même problème pour toutes les valeurs positives ou nulle du paramètre $\lambda$.

2.2. Forme courte du processus (pour une valeur donnée de $\lambda$ ).

On considère le système linéaire suivant :

\begin{tabular}{|c|c|}
\hline$\underset{(n, n)}{C} x+\underset{(n, m)}{A^{\prime}} u-\underset{(n, n)}{I v}$ & $+\underset{(n, n)}{E z} z=-\lambda \underset{(n, 1)}{p}$ \\
\hline$\underset{(m, n)}{A} x$ & $+\underset{(m, 1)}{w}=\underset{(m, 1)}{b}$ \\
\hline$x \geqq 0$ & $v \geqq 0, w \geqq 0, \quad z \geqq 0$ \\
\hline
\end{tabular}

où $\underset{(n, n)}{I}$ est une matrice unité, $\underset{(n, n)}{E}$ est une matrice diagonale dont les éléments diagonaux $e_{1}, e_{2}, \ldots, e_{n}$, égaux à 1 ou à -1 , ne seront fixés qu'à la fin de la première phase du processus (c'est-à-dire après l'annulation de $w$ ).

Les coordonnées de $\underset{(m, 1)}{w}$ et de $\underset{(n, 1)}{z}$ sont des variables artificielles.

On suppose $\underset{(m, 1)}{b} \geqq 0$, ce qui ne nuit pas à la généralité. 
$\mathbf{1}^{\mathrm{re}}$ phase. - On part du programme de base

$$
\left\{\begin{array} { c } 
{ E z = - \lambda p , } \\
{ w = b , }
\end{array} \quad \left\{\begin{array}{c}
x=0 \\
u=0 \\
v=0
\end{array}\right.\right.
$$

et l'on applique la méthode du Simplexe pour minimiser la fonction $\sum_{i=1}^{m} w_{i}$, en tenant compte des conditions de signe $w \geqq 0$ et $x \geqq 0$, mais en laissant les $(m+n)$ variables $u_{i}$ et $v_{j}$ hors de la base (donc nulles), et les $n$ variables $e_{j} z_{j}$ dans la base sans se préoccuper de leurs signes (puisque les coefficients $e_{j}$ ne sont pas fixés).

Si les contraintes $\left\{\begin{array}{c}A x=b \\ x \geqq 0\end{array}\right\}$ du problème initial sont compatibles, ce que l'on suppose, cette première phase du processus conduit, par des échanges entre les $w_{i}$ et les $x_{j}$, à annuler les $m$ variables artificielles $w_{i}$, et à supprimer éventuellement des contraintes redondantes parmi les $m$ égalités $A x=b$, ainsi que les coordonnées correspondantes de $u$. $(m, 1)$

A la fin de cette première phase, on dispose d'un programme $x$ réalisable du $(n, 1)$ problème initial, $w$ est nul et peut être supprimé, et la base est constituée par $(m, 1)$ certaines variables $x_{j}$ (en nombre égal au rang de A) et toutes les variables $z_{j}$. On choisit alors les coefficients $e_{j}$, égaux à 1 ou à-1, de telle manière que les valeurs des $n$ variables $z_{j}$ soient toutes positives ou nulles.

Pour simplifier l'écriture, on supposera que rang de $\underset{(m, n)}{A}=m$.

$2^{\mathrm{e}}$ phase. - A partir du programme de base ainsi obtenu, où $z \geqq 0$ et $v=0$, on applique la méthode du Simplexe pour minimiser la fonction $\sum_{j=1}^{n} z_{j}$, en tenant compte des conditions de signe $z \geqq 0, x \geqq 0$ et $v \geqq 0$, en faisant d'abord entrer dans la base, où elles resteront, les $m$ variables $u_{i}$ [de signes quelconques $\left({ }^{3}\right)$ ], et en s'interdisant, à chaque pas du processus, de faire entrer dans la base une variable $v_{j}$ $\left(\right.$ ou $\left.x_{j}\right)$ si la variable complémentaire $x_{j}\left(o u v_{j}\right) s^{\prime} y$ trouve déjà. Ainsi les relations d'exclusion $v_{j} x_{j}=0, \forall j$ sont-elles constamment vérifiées.

Alors, comme on l'établira au paragraphe 2.4.2,

- ou bien le processus conduit à annuler les $n$ variables artificielles $z_{j}$, et donne ainsi les solutions extrêmes du problème initial (et de ses conditions de Kuhn et Tucker);

$\left({ }^{3}\right)$ On pourrait supprimer, dans les tableaux de calcul ultérieurs, les lignes relatives aux $m$ variables $u_{i}$. 
- ou bien le processus se termine pour une valeur non nulle de $\sum_{j=1}^{n} z_{j}$, et conduit à montrer que $f(\lambda, x)$ n'a pas de minimum fini dans le problème initial.

Dans le cas où $\left\{\begin{array}{l}C r=0 \\ A r=0\end{array}\right\} \Rightarrow[r=0]$, et en particulier si $x^{\prime} C x>0, \forall x \neq 0$, on établira que $z=0$ à la fin du processus, de sorte que le problème initial admet une solution unique (cf. $\S 1.1)$.

2.3. Forme longue du processus (pour $\lambda$ croissant à partir de 0 )

On considère le système linéaire suivant :

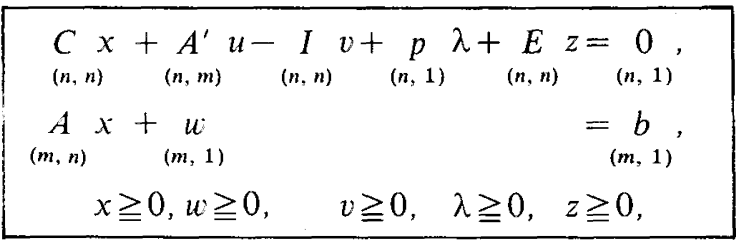

où les notations sont celles qui ont été définies au paragraphe 2.2 à propos de la forme courte, et où l'on suppose encore $b \geqq 0$.

$\mathbf{1}^{\text {re }}$ et $2^{\mathrm{e}}$ phases. - On part du programme de base (dégénéré) :

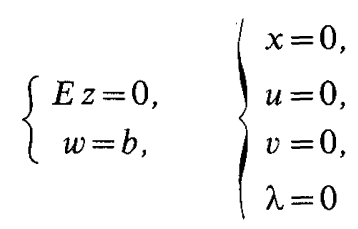

et l'on applique successivement les deux phases de la forme courte du processus pour $\lambda=0$, la variable $\lambda$ restant hors de la base.

A la fin de ces deux phases, on obtient les solutions extrêmes du problème initial et de ses conditions de Kuhn et Tucker pour la valeur 0 du paramètre $\lambda$. (Il est exclu que $f(0, x)=(1 / 2) x^{\prime} C x$ puisse décroître indéfiniment puisque $x^{\prime} C x \geqq 0, \forall x$.)

Toutes les variables artificielles $w_{i}$ et $z_{j}$ sont alors nulles et peuvent être supprimées.

vol. $13, \mathrm{n}^{\circ} 2$, mai 1979 
Pour simplifier l'écriture, on supposera que rang de $\underset{(m, n)}{A}=m$.

$\mathbf{3}^{\mathrm{e}}$ phase. - On part du (ou de l'un des) programme(s) de base ainsi obtenu(s) pour le système linéaire

$$
\left\{\begin{aligned}
& C x+A^{\prime} u-I v+p \lambda=0, \\
& A x=b, \\
& x \geqq 0, \quad v \geqq 0, \lambda \geqq 0,
\end{aligned}\right.
$$

programme dans lequel $\lambda=0$ ( $\lambda$ étant hors de la base) et $v_{j} x_{j}=0, \forall j$, et l'on applique la méthode du Simplexe pour maximiser $\lambda$ (ou minimiser $-\lambda$ ), sous ces contraintes linéaires, en cherchant d'abord à faire entrer $\lambda$ dans la base, et en $s^{\prime}$ interdisant, à chaque pas du processus, de faire entrer dans la base une variable $v_{j}$ (ou $x_{j}$ ) si la variable complémentaire $x_{j}\left(\right.$ ou $\left.v_{j}\right) s^{\prime} y$ trouve déjà, de sorte que $v_{j} x_{j}=0, \forall j$.

Alors, comme on l'établira au paragraphe 2.4.3,

- ou bien le processus conduit à faire croître indéfiniment $\lambda$ à partir de 0 , en passant par une suite finie de valeurs $0=\lambda_{0}<\lambda_{1}<\ldots<\lambda_{k-1}<\lambda_{k}$ (éventuellement réduite à $0=\lambda_{0}$ ), et donne la suite des solutions extrêmes correspondantes du problème initial (et de ses conditions de Kuhn et Tucker), ainsi qu'une direction d'infinitude pour $\lambda>\lambda_{k}$, à partir de quoi il est facile de déterminer les solutions de ce problème, par interpolation ou par extrapolation linéaire, pour toute valeur positive du paramètre $\lambda$;

- ou bien le processus se termine pour $\lambda=0$, et conduit à montrer que $f(\lambda, x)$ n'a pas de minimum fini dans le problème initial si $\lambda>0$.

Dans le cas où $\left\{\begin{array}{l}C r=0 \\ A r=0\end{array}\right\} \Rightarrow[r=0]$, et en particulier si $x^{\prime} C x>0, \forall x \neq 0$, on établira que le processus ne peut pas se terminer pour $\lambda=0$, de sorte que le problème initial (dont les contraintes sont supposées compatibles) admet une solution unique ( $c f . \S 1.1)$ pour toute valeur positive ou nulle du paramètre $\lambda$. (Pour chaque valeur de $\lambda$, cette solution peut être obtenue par la forme courte du processus.)

\section{4. Étude de l'efficacité de la méthode de Wolfe complétée}

2.4.1. ÉtUde GÉNÉRALE DES CAS DE « BLOCAGE » DU PROCESSUS

Soit le problème de programmation linéaire suivant :

R.A.I.R.O. Recherche opérationnelle/Operations Research 


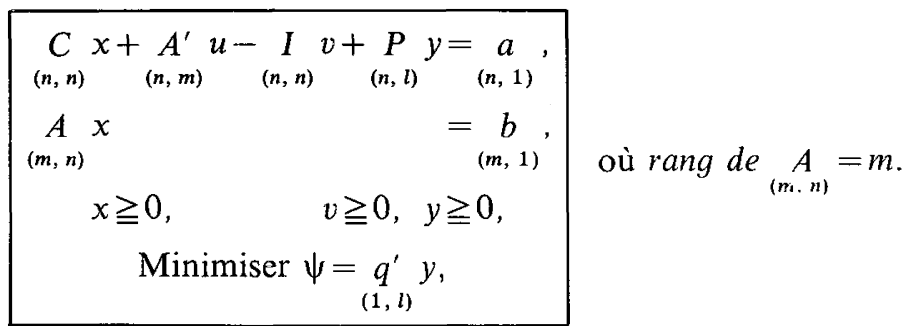

On cherche à résoudre ce programme linéaire par la méthode du Simplexe, en faisant d'abord entrer dans la base les $m$ variables $u_{i}$ (de signes quelconques) et en tenant compte de la restriction de Wolfe qui, pour assurer le respect des relations d'exclusion $v_{j} x_{j}=0, \forall j$, interdit de faire entrer dans la base une variable $v_{j}$ (ou $x_{j}$ ) si la variable complémentaire $x_{j}$ (ou $v_{j}$ ) s'y trouve déjà.

Soit un tableau du Simplexe conduisant à un « blocage », en ce sens que, compte tenu de la restriction de Wolfe, il est impossible de faire décroître $\psi=q^{\prime} y$ en faisant croitre à partir de 0 l'une des variables hors base qui peuvent entrer dans la base, ce qui se traduit par le fait que les coefficients de ces variables sont positifs ou nuls dans l'expression de $\psi$ en fonction des variables hors base.

Soit $(\bar{x}, \bar{u}, \bar{v}, \bar{y})$ le programme réalisable extrême correspondant à ce tableau, programme où la base ne contient aucun couple de variables complémentaires $v_{j}, x_{j}$, et soit la partition $V, W, X$ de l'ensemble d'indices $\{1,2, \ldots, n\}$ telle que

$\left\{\begin{array}{l}\bar{v}_{j} \text { est dans la base pour } j \in V \text {, hors de la base pour } j \in W \text { et pour } j \in X, \\ \bar{x}_{j} \text { est hors de la base pour } j \in V \text { et pour } j \in W \text {, dans la base pour } j \in X .\end{array}\right.$

Puisque $\operatorname{rang}$ de $A=m$, il y a $(m+n)$ variables de base, dont les $m$ variables $\bar{u}_{i}$, et, s'il y a au moins une variable $\bar{y}_{h}$ dans la base, card $V+$ card $X<n$ et $W \neq \emptyset$.

Le programme linéaire considéré et le programme dual s'écrivent :

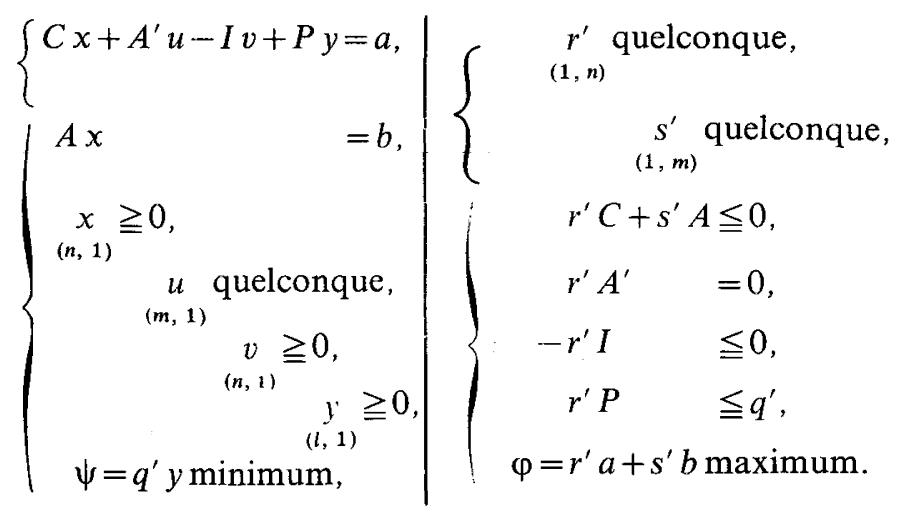


Soit $\left(\overline{r^{\prime}}, \overline{s^{\prime}}\right)$ le programme de base (en général irréalisable) associé, dans le dual, au programme extrême $(\bar{x}, \bar{u}, \bar{v}, \bar{y})$ et correspondant au même tableau du Simplexe. Ce couple de programmes de base associés vérifie les relations d'exclusion qui impliquent l'égalité des deux fonctions à optimiser : $\bar{\psi}=q^{\prime} \bar{y}=\bar{r}^{\prime} a+\bar{s}^{\prime} b=\bar{\varphi}$.

De plus, pour tout programme $(x, u, v, y)$ réalisable,

$$
\psi-\bar{\psi}=\psi-\bar{\varphi}=-\overline{r^{\prime}} a-\bar{s}^{\prime} b+q^{\prime} y=-\left(\overline{r^{\prime}} C+\overline{s^{\prime}} A\right) x-\overline{r^{\prime}} A^{\prime} u+\bar{r}^{\prime} v+\left(q^{\prime}-\overline{r^{\prime}} P\right) y,
$$

ce qui donne l'expression de $\psi$ en fonction des variables hors base (en vertu des relations d'exclusion entre $\left(\bar{r}^{\prime}, \bar{s}^{\prime}\right)$ et $\left.(\bar{x}, \bar{u}, \bar{v}, \bar{y})\right)$.

D'après l'hypothèse de "blocage " qui a été explicitée, et compte tenu de la structure du couple de programmes de base associés $(\bar{x}, \bar{u}, \bar{v}, \bar{y})$ et $\left(\bar{r}^{\prime}, \overline{s^{\prime}}\right)$,

\begin{tabular}{|lllll|}
\hline pour $j \in V$, & $\bar{x}_{j}=0$, & $\overline{r^{\prime}} C^{j}+\bar{s}^{\prime} A^{j} q c q$, & $\bar{v}_{j} \geqq 0$ (dans la base), & $\bar{r}_{j}=0$, \\
pour $j \in W$, & $\bar{x}_{j}=0$, & $\bar{r}^{\prime} C^{j}+\bar{s}^{\prime} A^{j} \leqq 0$, & $\bar{v}_{j}=0$, & $\bar{r}_{j} \geqq 0$, \\
pour $j \in X$, & $\bar{x}_{j} \geqq 0$ (dans la base); & $\overline{r^{\prime}} C^{j}+\bar{s}^{\prime} A^{j}=0$, & $\bar{v}_{j}=0$, & $\bar{r}_{j} q c q ;$ \\
& $\bar{u} q c q$ (dans la base), & $\overline{r^{\prime}} A^{\prime}=0$, & & \\
& $\bar{y} \geqq 0$, & $\bar{r}^{\prime} P \leqq q^{\prime}$, & $\overline{r^{\prime}} P \bar{y}=q^{\prime} \bar{y}=\bar{r}^{\prime} a+\bar{s}^{\prime} b$, & \\
\hline
\end{tabular}

car la restriction de Wolfe interdit de faire entrer dans la base $x_{j} \mid j \in V$ et $v_{j} \mid j \in X$, mais permet d'y faire entrer $x_{j} \mid j \in W$ ou $v_{j} \mid j \in W$, ou tout $y_{h}$ qui ne s'y trouve pas déjà.

Ces relations impliquent (compte tenu du fait que $x^{\prime} C x \geqq 0, \forall x$ ) :

$$
\begin{aligned}
& \left\{\begin{array}{c}
\left(\overline{r^{\prime}} C+\overline{s^{\prime}} A\right) \bar{r} \leqq 0 \\
A \bar{r}=0
\end{array}\right\} \Rightarrow\left[\overline{r^{\prime} C} \bar{r} \leqq 0\right] \Rightarrow\left[\overline{r^{\prime} C} \bar{r}=0\right] \Rightarrow[C \bar{r}=0], \\
& \left\{\begin{array}{c}
\left(\overline{r^{\prime}} C+\overline{s^{\prime}} A\right) \bar{x}=0 \\
\overline{r^{\prime}} C=0
\end{array}\right\} \Rightarrow\left[\overline{s^{\prime}} A \bar{x}=0\right] \quad \Rightarrow \quad\left[\overline{s^{\prime}} b=0\right] \text {. }
\end{aligned}
$$

De plus

$\underset{(1, m)}{\left[\overline{s^{\prime}} A^{X}=0\right]} \Rightarrow\left[\overline{s^{\prime}}=0\right], \quad$ puisque rang de $A^{X}=\operatorname{rang}$ de $A=m$.

Ainsi, dans les conditions de «blocage » envisagées, il existe un vecteur $\bar{r}$ tel que

$$
\begin{aligned}
\left\{\begin{array} { c } 
{ C } \\
{ ( n , n ) } \\
{ \underset { ( m , n ) } { A } \overline { r } = 0 , }
\end{array} \quad \left\{\begin{array}{l}
\bar{r}_{V}=0 \\
\bar{r}_{W} \geqq 0 \\
\underset{(l, n)}{P^{\prime}} \bar{r} \leqq \underset{(l, 1)}{q}, \quad \underset{(1, n)}{a^{\prime}} \bar{r}=\underset{(1, l)}{q^{\prime}} \bar{y}
\end{array}\right.\right.
\end{aligned}
$$

R.A.I.R.O. Recherche opérationnelle/Operations Research 
et

$$
\psi-\bar{\psi}=\overline{r^{\prime}} v+\left(q^{\prime}-\bar{r}^{\prime} P\right) y,
$$

pour tout programme $(x, u, v, y)$ réalisable.

Si $q^{\prime} \bar{y} \neq 0$, ou si $q \geq 0, \bar{r} \neq 0$. Il existe alors, en général, d'autres programmes réalisables $(x, u, v, y)$ donnant la même valeur $\bar{\psi}$ à $\psi=q^{\prime} y$, soit $(\bar{x}+t \bar{r}, \bar{u}, \bar{v}, \bar{y})$ pour tout scalaire $t$ tel que $\bar{r}_{W} \geqq 0$ et $\bar{x}_{X}+t \bar{r}_{X} \geqq 0$. Ces programmes vérifient les relations d'exclusion $\bar{v}_{j}\left(\bar{x}_{j}+t \bar{r}_{j}\right)=0, \forall j$.

S'il y a au moins une variable $\bar{y}_{h}$ dans la base, $W \neq \emptyset$ et, puisque le programme $(\bar{x}, \bar{u}, \bar{v}, \bar{y})$ est extrême, $\bar{r}_{W} \neq 0$ de sorte que $t \bar{r}_{W} \geqq 0 \Rightarrow t \geqq 0$. En effet, si $\bar{r}_{W}$ était nul, la condition $\bar{x}_{X}+t \bar{r}_{X} \geqq 0$ permettrait au scalaire $t$ de varier dans un intervalle encadrant 0 , du moins dans le cas général où $\bar{x}_{X}>0$ (on peut toujours se ramener à ce cas par l'un des procédés qui permettent d'éviter tout risque de « cyclage » dans l'application de la méthode du Simplexe).

Alors $\quad P^{\prime}(\bar{x}+t \bar{r}) \leqq P^{\prime} \bar{x}+t q$ et $a^{\prime}(\bar{x}+t \bar{r})=a^{\prime} \bar{x}+t q^{\prime} \bar{y}$.

- Si $\bar{r}_{X} \geqq 0$, le scalaire $t$ peut croître indéfiniment à partir de 0 .

- Si $\bar{r}_{X} \geq 0$, le scalaire $t$ peut croître de 0 à un maximum

$$
\theta=\min \left\{-\frac{\bar{x}_{j}}{\bar{r}_{j}} \mid j \in X \text { et } \bar{r}_{j}<0\right\} .
$$

Dans le second cas, où $\bar{r}_{j}$ n'est pas positif ou nul pour tout $j \in X$, il existe donc un programme réalisable $(x, u, v, y)=(\bar{x}+\theta \bar{r}, \bar{u}, \bar{v}, \bar{y})$ dans lequel

$$
\begin{cases}x_{V}=\bar{x}_{V}=0, & v_{V}=\bar{v}_{V} \geqq 0, \\ x_{W}=\theta \bar{r}_{W} \geqq 0, & v_{W}=\bar{v}_{W}=0, \\ x_{X}=\bar{x}_{X}+\theta \bar{r}_{X} \geqq 0, & v_{X}=\bar{v}_{X}=0, \\ y_{Y}=\bar{y}_{Y} \geqq 0, & y_{\bar{Y}}=\bar{y}_{\bar{Y}}=0\end{cases}
$$

(si $\bar{y}_{h}$ est dans la base pour $h \in Y$, hors de la base pour $h \in \bar{Y}$ ).

Mais le passage de $(\bar{x}, \bar{u}, \bar{v}, \bar{y})$ à $(\bar{x}+\theta \bar{r}, \bar{u}, \bar{v}, \bar{y})$ ne correspond pas à un pas de la méthode du Simplexe si $\bar{r}_{W}$ a plusieurs coordonnées strictement positives, car plusieurs variables hors base $x_{j}$ telles que $j \in W$ et $\overline{r_{j}}>0$ prennent alors des valeurs strictement positives tandis qu'une variable de base au moins s'annule.

$x_{j}$ telle que $j \in X, \overline{r_{j}}<0$ et $\overline{x_{j}} /\left|\overline{r_{j}}\right|$ minimum parmi les rapports analogues.

Cependant, il existe toujours un programme extrême dans lequel $x_{V}=0, v_{W}=0$, $v_{X}=0, y_{Y}=0$ et $x_{j}=0$, et, à partir du tableau du Simplexe correspondant à $(\bar{x}, \bar{u}, \bar{v}, \bar{y})$, ce programme extrême peut être atteint par un ou plusieurs pas de la méthode du Simplexe.

vol. $13, \mathrm{n}^{\circ} 2$, mai 1979 


\begin{tabular}{c|ccccc|c} 
& $x_{W}^{\prime}$ & $x_{V}^{\prime}$ & $v_{W}^{\prime}$ & $v_{X}^{\prime}$ & $y_{\bar{Y}}^{\prime}$ & \\
\hline$\psi$ & 0 & 0 & $-\overline{r_{W}^{\prime}}$ & $-\overline{r_{X}^{\prime}}$ & $-\left(q^{\prime}-\overline{r^{\prime}} P\right)_{Y}$ & $\bar{\psi}$ \\
\hline$x_{X}$ & & & & & & $\bar{x}_{X}$ \\
$v_{V}$ & & & & & $\bar{v}_{V}$ \\
$y_{Y}$ & & & & & & $\bar{y}_{Y}$ \\
$u$ & & & & & & $\bar{u}$
\end{tabular}

A chacun de ces pas, $x_{V}, v_{W}, v_{X}, y_{Y}$ restent hors de la base, tandis que des échanges se produisent entre une variable hors base et une variable de base dans l'ensemble des variables appartenant à $x_{W}, x_{X}, v_{V}, y_{Y}$. Chacun de ces échanges respecte la restriction de Wolfe et ne modifie pas la valeur de la fonction $\psi$ ni son expression en fonction de $v$ et $y$.

Pour éviter une exploration aléatoire de l'ensemble des programmes extrêmes où $x_{V}=0, v_{W}=0, v_{X}=0, y_{\bar{Y}}=0$, on peut appliquer à cette exploration la méthode $\mathrm{du}$ Simplexe en se proposant de minimiser la variable $x_{\underline{j}}$, qu'il est facile de déterminer à partir du tableau ci-dessus où figurent les $\bar{x}_{j}$ et les $\bar{r}_{j}$ pour $j \in X$.

On est assuré de pouvoir faire sortir $x_{j}$ de la base à la fin de cette exploration, et le tableau du Simplexe ainsi obtenu ne conduit pas à un blocage, puisque $x_{\underline{j}}$ et $v_{\underline{j}}$ sont alors hors de la base et que $r_{j}<0$. La restriction de Wolfe permet donc de faire entrer $v_{j}$ dans la base, et la croissance de $v_{j}$ à partir de 0 fait décroître

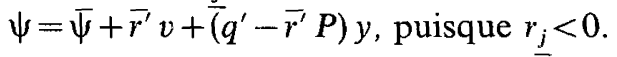

(Pour être sûr d'obtenir ainsi une décroissance stricte de la fonction $\psi$, on peut éventuellement utiliser l'un des procédés qui permettent d'éviter tout risque de « cyclage » dans l'application de la méthode du Simplexe.)

\subsubsection{Application a La FORME COURTE Du processus}

Le phénomène de « blocage » qui vient d'être étudié peut $a$ priori se présenter dans la $2^{\mathrm{e}}$ phase du processus sous forme courte, et les résultats obtenus au paragraphe 2.4 .1 peuvent être appliqués à ce cas avec les notations suivantes :

$$
\underset{(n, l)}{P}=\underset{(n, n)}{E}, \quad \underset{(l, 1)}{y}=\underset{(n, 1)}{z}, \quad \underset{(n, 1)}{a}=-\lambda \underset{(n, 1)}{p}, \quad \underset{(1, l)}{q^{\prime}}=(1,1, \ldots, 1) .
$$

Ainsi, dans les conditions de « blocage » envisagées, il existe un vecteur $\bar{r}$ tel que

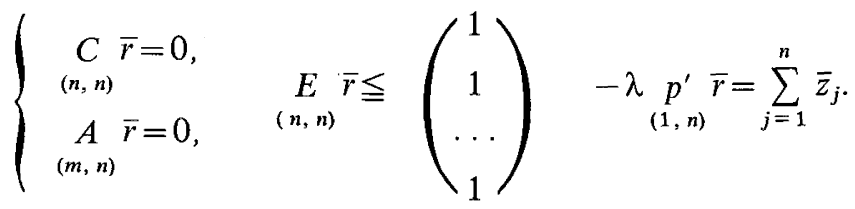

R.A.I.R.O. Recherche opérationnelle/Operations Research 
1) Si $\lambda p^{\prime} \bar{r}=0, \sum_{j=1}^{n} \bar{z}_{j}=0$, ce qui implique $\bar{z}_{j}=0, \forall j$. Toutes les variables artificielles sont alors nulles, et le tableau du Simplexe considéré donne une solution extrême du problème initial (et de ses conditions de Kuhn et Tucker). Si cette solution n'est pas unique, le tableau présente une dégénérescence qui permet de déterminer toutes les solutions en poursuivant le processus, compte tenu des propriétés établies au paragraphe 1.3. Le problème initial est ainsi résolu.

Telle est, en particulier, la fin du processus si $\lambda=0$, ou si $\left\{\begin{array}{l}C r=0 \\ A r=0\end{array}\right\} \Rightarrow[r=0]$, ce qui est le cas si la forme quadratique $x^{\prime} C x$ est définie positive (c'est-à-dire strictement positive pour tout $x \neq 0$ ). Dans ce dernier cas, auquel on peut toujours se ramener par la méthode des perturbations (voir $\S 1.2$ ), la solution du problème initial est unique (voir § 1.1).

2) Si, au contraire, $\sum_{j=1}^{n} \bar{z}_{j}>0$ au moment $d u$ « blocage » (ce qui n'est possible que si $\lambda>0$ et $x^{\prime} C x$ semi-définie positive), $\bar{r} \neq 0$ et il existe en général un ensemble infini de programmes réalisables $(\bar{x}+t \bar{r}, \bar{u}, \bar{v}, \bar{z})$, donnant la même valeur à $\sum_{j=1}^{n} z_{j}$ et vérifiant les relations d'exclusion $\bar{v}_{j}\left(\bar{x}_{j}+t \bar{r}_{j}\right)=0, \forall j$.

La fonction à minimiser dans le problème initial y prend les valeurs

$$
f(\lambda, \bar{x}+t \bar{r})=\lambda p^{\prime}(\bar{x}+t \bar{r})+\frac{1}{2} \bar{x}^{\prime} \dot{C} \bar{x}=f(\lambda, \bar{x})-t \sum_{j=1}^{n} \bar{z}_{j},
$$

car

$$
C \bar{r}=0 \quad \text { et } \quad \lambda p^{\prime} \bar{r}=-\sum_{j=1}^{n} \bar{z}_{j}<0 .
$$

Deux cas sont alors possibles, d'après l'étude faite au paragraphe 2.4.1:

- ou bien le scalaire t peut croître indéfiniment à partir de 0 (cas où $\bar{r}_{X} \geqq 0$ ), et $f(\lambda, \bar{x}+t \bar{r}) \rightarrow-\infty$ quand $t \rightarrow+\infty$, de sorte que la fonction convexe $f(\lambda, x) n^{\prime} a$ pas de minimum fini dans le problème initial;

- ou bien le scalaire t peut crô̂tre de 0 à un maximum

$$
\theta=\min \left\{-\frac{\bar{x}_{j}}{\bar{r}_{j}} \mid j \in X \text { et } \bar{r}_{j}<0\right\} \quad \text { (cas où } \bar{r}_{X} \geq 0 \text { ), }
$$

et il est possible de profiter des dégénérescences qui accompagnent le « blocage » pour atteindre, en poursuivant le processus, un programme extrême donnant la même valeur à $\sum_{j=1}^{n} z_{j}$, mais tel que le tableau du Simplexe correspondant ne soit vol. $13, \mathrm{n}^{\circ} 2$, mai 1979 
plus " bloqué » (c'est sur ce point que la méthode ici présentée complète la méthode de Wolfe).

Ainsi arrivera-t-on toujours, en un nombre fini de pas, soit à résoudre le problème initial, soit à montrer que $f(\lambda, x)$ n'a pas de minimum fini dans ce problème.

\subsubsection{Application a la forme longue du processus}

Le phénomène de « blocage " peut a priori se présenter, non dans la $2^{\mathrm{e}}$ phase où $\lambda=0$ (voir $\S 2.4 .2$ ), mais dans la $3^{\mathrm{e}}$ phase du processus sous forme longue, et les résultats obtenus au paragraphe 2.4 .1 peuvent être appliqués à ce cas avec les notations suivantes :

$$
\underset{(n, l)}{P}=\underset{(n, 1)}{p}, \quad \underset{(l, 1)}{y}=\underset{(1,1)}{\lambda}, \quad \underset{(n, 1)}{a}=\underset{(n, 1)}{0}, \quad \underset{(1, l)}{q^{\prime}}=-1 .
$$

Ainsi, dans les conditions de « blocage 》 envisagées, il existe un vecteur $\bar{r}$ tel que

$$
\left\{\begin{array}{l}
\underset{(n, n)}{C} \bar{r}=0, \\
\underset{(m, n)}{A} \bar{r}=0, \\
(1, n)
\end{array} \quad p^{\prime} \bar{r} \leqq-1, \quad 0=-\bar{\lambda} .\right.
$$

Donc, ou bien le « blocage » envisagé ne se produit pas, ou bien il se produit pour $\lambda=0$.

1) S'il ne se produit pas de " blocage », le processus de maximisation de $\lambda$, sous les conditions assurant le respect des relations d'exclusion $v_{j} x_{j}=0, \forall j$, conduit à faire croître indéfiniment $\lambda$ à partir de 0 , en passant par une suite finie de valeurs $0=\lambda_{0}<\lambda_{1}<\ldots<\lambda_{k-1}<\lambda_{k}$ (éventuellement réduite à $0=\lambda_{0}$ ), et donne la suite des solutions extrêmes correspondantes du problème initial (et de ses conditions de Kuhn et Tucker), soit $\left(x^{0}, u^{0}, v^{0}\right),\left(x^{1}, u^{1}, v^{1}\right), \ldots,\left(x^{k}, u^{k}, v^{k}\right)$, complétée par $\left(\xi^{k}, \alpha^{k}, \beta^{k}\right)$ tel que, pour tout scalaire $t \geqq 0,\left(x^{k}+t \xi^{k}, u^{k}+t \alpha^{k}, v^{k}+t \beta^{k}\right)$ soit solution du problème initial pour $\lambda=\lambda_{k}+t$.

En l'absence de dégénérescence, ces suites sont déterminées sans ambiguïté, et c'est ce que l'on suppose ici. Il en est certainement ainsi si $\left\{\begin{array}{l}C z=0 \\ A z=0\end{array}\right\} \Rightarrow[z=0]$, et en particulier si la forme quadratique $x^{\prime} C x$ est définie positive. (S'il en était autrement, le processus permettrait encore de déterminer toutes les solutions extrêmes, nécessairement en nombre fini.)

La restriction qui interdit, à chaque pas du processus, de faire entrer dans la base une variable $v_{j}$ (ou $x_{j}$ ) si la variable complémentaire $x_{j}$ (ou $v_{j}$ ) s'y trouve déjà implique :

$$
\left\{\begin{array}{l}
0=v^{\prime h} x^{h}=v^{\prime h} x^{h+1}=v^{\prime h+1} x^{h}=v^{\prime h+1} x^{h+1}, \quad \forall h \in\{0,1,2, \ldots, k-1\}, \\
0=v^{\prime k} x^{k}=v^{\prime k} \xi^{k}=\beta^{\prime k} x^{k}=\beta^{\prime k} \xi^{k} .
\end{array}\right.
$$

R.A.I.R.O. Recherche opérationnelle/Operations Research 
Les conditions de Kuhn et Tucker (linéaires si l'on excepte les relations d'exclusion) montrent alors que le problème initial, dépendant du paramètre $\lambda \geqq 0$, admet comme solutions :

$$
\begin{aligned}
& \text { pour } \lambda \in\left[\lambda_{h}, \lambda_{h+1}\right] \text {, } \\
& \qquad(x, u, v)=\frac{\lambda_{h+1}-\lambda}{\lambda_{h+1}-\lambda_{h}}\left(x^{h}, u^{h}, v^{h}\right)+\frac{\lambda-\lambda_{h}}{\lambda_{h+1}-\lambda_{h}}\left(x^{h+1}, u^{h+1}, v^{h+1}\right),
\end{aligned}
$$

pour $\lambda \geqq \lambda_{k}$,

$$
(x, u, v)=\left(x^{k}, u^{k}, v^{k}\right)+\left(\lambda-\lambda_{k}\right)\left(\xi^{k}, \alpha^{k}, \beta^{k}\right) .
$$

Ces solutions s'obtiennent par interpolation linéaire entre deux solutions extrêmes adjacentes, et par extrapolation linéaire à partir de $\left(x^{k}, u^{k}, v^{k}\right)$ dans la direction d'infinitude $\left(\xi^{k}, \alpha^{k}, \beta^{k}\right)$.

Le problème paramétrique initial est ainsi résolu pour tout $\lambda \geqq 0$, lorsqu'il ne se produit pas de «blocage ». Telle est, en particulier, la fin du processus si $\left\{\begin{array}{l}C r=0 \\ A r=0\end{array}\right\} \Rightarrow[r=0]$ (puisqu'il est alors impossible que $p^{\prime} \bar{r} \leqq-1$ ), ce qui est le cas si la forme quadratique $x^{\prime} C x$ est définie positive.

2) S'il se produit un « blocage " pour $\lambda=0$ (ce qui n'est possible que si $x^{\prime} C x$ est semi-définie positive), cette situation se présente après que $\lambda$ est entré dans la base avec la valeur 0 , de sorte que card $W=1$. Alors $\bar{r} \neq 0$ et il existe en général un ensemble infini de programmes $(\bar{x}+t \bar{r}, \bar{u}, \bar{v})$ optimaux dans le problème initial pour $\lambda=0$.

La fonction à minimiser dans le problème initial y prend les valeurs

$$
f(\lambda, \bar{x}+t \bar{r})=\lambda p^{\prime}(\bar{x}+t \bar{r})+\frac{1}{2} \bar{x}^{\prime} C \bar{x} \leqq f(\lambda, \bar{x})-\lambda t,
$$

pour $\lambda \geqq 0$ et $t \geqq 0$, car $C \bar{r}=0$ et $p^{\prime} \bar{r} \leqq-1$.

Deux cas sont alors possibles, d'après l'étude faite au paragraphe 2.4.1:

- ou bien le scalaire t peut croître indéfiniment à partir de 0 (cas où $\bar{r}_{X} \geqq 0$ ), et, si $\lambda>0, f(\lambda, \hat{x}+t \hat{r}) \rightarrow-\infty$ quand $t \rightarrow+\infty$, de sorte que la fonction convexe $f(\lambda, x)$ n'a pas de minimum fini dans le problème initial dès que $\lambda>0$;

- ou bien le scalaire t peut croître de 0 à un maximum

$$
\theta=\min \left\{-\frac{\bar{x}_{j}}{\overline{\bar{r}}_{j}} \mid j \in X \text { et } \bar{r}_{j}<0\right\} \quad \text { (cas où } \bar{r}_{X} \geq 0 \text { ), }
$$

et le passage de $(\bar{x}, \bar{u}, \bar{v}) \grave{a}(\bar{x}+\theta \bar{r}, \bar{u}, \bar{v})$ correspond ici à un pas de la méthode du Simplexe, avec échange entre l'unique variable $x_{j} \mid j \in W$ (qui entre dans la base) et une variable $x_{j} \mid j \in X, \bar{r}_{j}<0$ et $\bar{x}_{j}+\theta \bar{r}_{j}=0$ (qui sort de la base). Le tableau du vol. $13, \mathrm{n}^{\circ} 2$, mai 1979 
Simplexe ainsi obtenu, adjacent au précédent, donne encore la valeur 0 au paramètre $\lambda$, mais il n'est plus « bloqué ».

Ainsi arrivera-t-on toujours, en un nombre fini de pas, soit à faire croître indéfiniment $\lambda$ à partir de 0 et à résoudre le problème paramétrique initial pour tout $\lambda \geqq 0$, soit à montrer que $f(\lambda, x)$ n'a pas de minimum fini dans ce problème dès que $\lambda>0$.

\subsection{Note sur le calcul de $f(\lambda, x)$ dans la méthode de Wolfe}

D'après le calcul fait au paragraphe 1.3 , et compte tenu des variables artificielles, coordonnées de $w$ et de $z$, introduites dans les conditions de Kuhn et Tucker ( $c f . \S 2.2)$, $(m, 1) \quad(n, 1)$

$$
2 f(\lambda, x)=\lambda p^{\prime} x-u^{\prime} b+v^{\prime} x-E z^{\prime} x+u^{\prime} w \text {. }
$$

A la fin de la $1^{\text {re }}$ phase du processus, $w=0$, et à la fin de la $2^{\mathrm{e}}$ phase, $z=0$. De plus, la restriction de Wolfe impose constamment $v^{\prime} x=0$.

$$
(m, 1)
$$

Il est donc intéressant d'ajouter une ligne dans les tableaux $d u$ Simplexe pour calculer, au cours du processus, l'expression $\lambda p^{\prime} x-u^{\prime} b$, linéaire en $x$ et $u^{\prime \prime}$, qui donnera, à la fin du processus, la valeur optimale de $2 f(\lambda, x) \stackrel{(n, 1)}{(\text { quand }} \stackrel{(1, m)}{\text { elle }}$ existe).

Dans la forme longue du processus, $\left(\lambda p^{\prime} x-u^{\prime} b\right)$ dépend linéairement du paramètre $\lambda$ dans chaque tableau du Simplexe et, au cours de la $3^{\mathrm{e}}$ phase, les calculs d'interpolation et d'extrapolation linéaires doivent être effectués à partir de ces expressions linéaires en $\lambda$, sans que $\lambda$ soit remplacé par sa valeur dans chaque tableau. Ainsi, quand le minimum def $(\lambda, x)$ existe pour tout $\lambda \geqq 0$, c'est une fonction concave de $\lambda$ (cf. §1.1), quadratique par morceaux.

\section{MÉTHOdE DE DANTZIG}

\subsection{Principe $\left({ }^{4}\right)$}

On considère le problème non paramétrique de programmation convexe à contraintes linéaires, qui coïncide avec celui du paragraphe 1 pour $\lambda=1$ :

$$
\text { Minimiser } f(x)=\underset{(1, n)}{p^{\prime}} x+\frac{1}{2} x^{\prime} \underset{(n, n)}{C} x,
$$

sous les contraintes :

$$
\underset{(m, n)}{A} x=\underset{(m, 1)}{b} \quad \text { et } \quad x \geqq \underset{(n, 1)}{0},
$$

$\left({ }^{4}\right)$ Pour faciliter la lecture, on rappelle d'abord ici les résultats obtenus au paragraphe 1.3. 
$C$ étant une matrice symétrique réelle telle que $x^{\prime} \quad C \quad x \geqq 0, \forall x \in \mathbf{R}^{n}$. $(n, n)$ $(1, n)(n, n)(n, 1)$

Le lagrangien (formé sans tenir compte des conditions de signe $x \geqq 0$ ) :

$$
l(x, u)=\underset{(1, n) .}{p^{\prime}} x+\frac{1}{2} x^{\prime} \underset{(n, n)}{C} x+\underset{(1, m)}{u^{\prime}}(\underset{(m, n)}{A} x-\underset{(m, 1)}{b})
$$

est égal à la fonction économique $f(x)$ pour tout programme $x$ réalisable, et les conditions de Kuhn et Tucker, nécessaires et suffisantes ( $c f . \S 1.3$ ), s'écrivent :

$$
\begin{gathered}
\nabla_{x}^{\prime} l(x, u)=\underset{(n, 1)}{p}+\underset{(n, n)}{C} x+\underset{(n, m)}{A^{\prime}} u=\underset{(n, 1)}{v}(\geqq 0), \\
-\underset{(m, 1)}{b+\underset{(m, n)}{A} x} x=0, \\
x \geqq 0, \quad v \geqq 0, \quad v_{j} x_{j}=0, \quad \forall j .
\end{gathered}
$$

Compte tenu des $(n+m)$ égalités précédentes, linéaires en $x_{j}, u_{i}, v_{j}$,

$$
2 f(x)=p^{\prime} x-u^{\prime} b+v^{\prime} x .
$$

S'il existe une solution des conditions de Kuhn et Tucker pour laquelle $x_{j}>0$ (ou $v_{j}>0$ ), alors dans toute solution de ces solutions $v_{j}=0$ (ou $x_{j}=0$ ) (cf. $\S 1.3)$.

L'ensemble des points $(\bar{x}, \bar{u}, \bar{v})$ solutions des conditions de Kuhn et Tucker est donc un tronçon de l'espace $\mathbf{R}^{m+2 n}$, de sorte qu'il peut être défini à partir de ses points extrêmes, et éventuellement de ses directions d'infinitude extrêmes.

C'est ce qui conduit à chercher des solutions de base (au sens de la méthode du Simplexe) du système des conditions linéaires de Kuhn et Tucker $(m+n$ égalités et $2 n$ conditions de signe portant sur $m+2 n$ variables), en leur imposant de vérifier les $n$ relations d'exclusion $v_{j} x_{j}=0, \forall j$.

Dans la méthode de Dantzig, on y parvient en appliquant à ce système linéaire une méthode analogue à la méthode du Simplexe, de telle manière que les variables $x_{j}$ soient constamment positives ou nulles tandis que les variables $v_{j}$ ne sont toutes positives ou nulles que si l'optimum est atteint. Les programmes de base successifs vérifient soit toutes les relations $v_{j} x_{j}=0$ (programmes dits "standard»), soit toutes ces relations sauf une (programmes dits "non standard $)$ ). De plus, les passages de programme de base à programme de base adjacent, selon l'algorithme de Gauss, sont organisés de telle manière que le lagrangien $l(x)$, constamment égal à la fonction économique $f(x)$ à minimiser, ne puisse pas croître. Et, pourvu que l'éventualité d'un cyclage soit écartée, le processus conduit, en un nombre fini de pas, soit aux solutions extrêmes du problème posé (et de ses conditions de Kuhn et Tucker), soit à montrer que $f(x)$ n'a pas de minimum fini dans ce problème. 


\subsection{Description de l'algorithme}

Un programme de base est dit «standard 》 si la base utilisée comprend une variable et une seule dans chaque couple de variables complémentaires $\left(v_{j}, x_{j}\right)$. Un tel programme vérifie toutes les relations d'exclusion $v_{j} x_{j}=0$.

Un programme de base est dit "non standard " si la base utilisée comprend exactement un couple de variables complémentaires, de telle manière qu'il y ait aussi exactement un couple de variables complémentaires hors de la base. Un tel programme vérifie toutes les relations d'exclusion $v_{j} x_{j}=0$ sauf une.

$\mathbf{1}^{\mathrm{re}}$ phase. - On détermine un programme standard de départ, dans lequel toutes les variables $x_{j}$ sont positives ou nulles, les variables $v_{j}$ étant de signes quelconques.

Si un tel programme de départ n'est pas en évidence, on considère le système linéaire suivant :

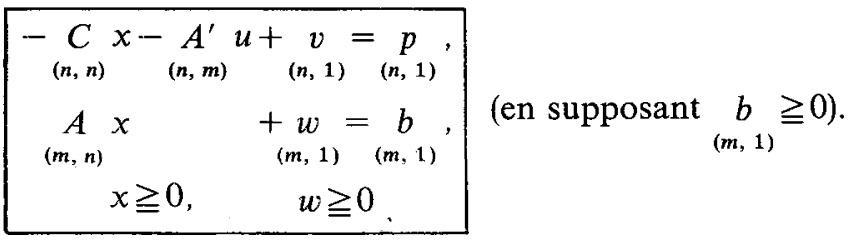

Les coordonnées de $\underset{(m, 1)}{w}$ sont des variables artificielles.

On part du programme de base

$$
\begin{cases}v=p, & x=0 \\ w=b, & u=0\end{cases}
$$

et l'on applique la méthode du Simplexe pour minimiser la fonction $\sum_{i=1}^{m} w_{i}$ par des échanges entre les $w_{i}$ et les $x_{j}$. Si les contraintes $\left\{\begin{array}{c}A x=b \\ x \geqq 0\end{array}\right\}$ du problème posé sont compatibles, ce que l'on suppose, on est ainsi conduit à annuler les $m$ variables artificielles $w_{i}$, et à supprimer éventuellement des contraintes redondantes parmi les $m$ égalités $A x=b$, ainsi que les coordonnées correspondantes de $u$.

Pour assurer le respect des relations d'exclusion $v_{j} x_{j}=0, \forall j$ à la fin de cette première phase, il faut faire sortir de la base les variables $v_{j}$ complémentaires des variables $x_{j} q u i$ sont entrées dans la base. Il suffit pour y parvenir d'échanger ces variables $v_{j}$ avec les variables $u_{i}$ (de signes quelconques), ce qui est toujours 
possible compte tenu de la forme du système linéaire considéré. On peut par exemple échanger $u_{h}$ et $v_{k}$ aussitôt après avoir échangé $w_{h}$ et $x_{k}$ (et, éventuellement, $v_{k}$ et $v_{t}$ aussitôt après avoir échangé $x_{k}$ et $x_{l}$ ). Il est facile de vérifier que, si l'on procède ainsi systématiquement, les deux pivots correspondant à ces deux échanges successifs sont opposés.

Pour simplifier l'écriture, on supposera que rang de $\underset{(m, n)}{A}=m$.

On dispose alors, à la fin de la première phase, d'un programme standard de départ dans lequel, les $m$ variables artificielles $w_{i}$ ayant été supprimées, la base est constituée par $n$ variables $x_{j} \geqq 0$ ou $v_{j}$ de signes quelconques (une et une seule dans chaque couple $\left.\left(v_{j}, x_{j}\right)\right)$ et les $m$ variables $u_{i}$ de signes quelconques (qui resteront ensuite dans la base) $\left({ }^{5}\right)$.

$\mathbf{2}^{\mathrm{e}}$ phase. - On part du programme de base ainsi obtenu pour le système linéaire

$$
\left\{\begin{array}{l}
-C x-A^{\prime} u+v=p, \\
A x=b, \\
x \geqq 0,
\end{array}\right.
$$

programme dans lequel $v_{j} x_{j}=\dot{0}, \forall j$, puisqu'il s'agit d'un programme standard.

Si de plus $v \geqq 0$, ce programme est optimal, et le processus se termine aussitôt. Si le problème donné (avec ses conditions de Kuhn et Tucker) admet d'autres solutions, le tableau de calcul présente une dégénérescence qui permet de déterminer toutes les solutions, compte tenu des propriétés établies au paragraphe 1.3.

Si au contraire $v \geq 0$, le processus se poursuit par des passages de programme de base à programme de base adjacent, selon l'algorithme de Gauss, comme dans la méthode du Simplexe. Ces passages sont organisés de la façon suivante :

1) A PARTir D'Un PRogramme de base Standard ou $\exists j \mid v_{j}<0$

- on fait entrer dans la base en croissant une des variables hors base $x_{j}$ dont la variable complémentaire $v_{j}=\partial l(x, u) / \partial x_{j}$ (qui est dans la base) a une valeur négative (par exemple la valeur négative de plus grande valeur absolue), soit $x_{h} \mid v_{h}<0$;

- on fait sortir de la base celle des variables de base $x_{j}$ et de la variable $v_{h}$, complémentaire de la variable entrante $x_{h}$, qui s'annule la première quand $x_{h}$ croît à partir de 0 (on montrera au paragraphe 3.3 qu'alors $v_{h}$ ne décroît pas). En cas d'ambiguité entre $v_{h}$ et une variable $x_{j}$, on choisit $v_{h}$ comme variable sortante.

$\left({ }^{5}\right)$ On pourrait supprimer, dans les tableaux de calcul ultérieurs, les lignes relatives aux $m$ variables $u_{i}$.

vol. $13, \mathrm{n}^{\circ} 2$, mai 1979 
Ainsi est-on assuré que, dans le passage au programme de base adjacent,

- toutes les variables $x_{j}$ restent positives ou nulles,

- la fonction $f(x)$ à minimiser décroît (sauf dans le cas de dégénérescence où $x_{h}$ resterait nulle en entrant dans la base), puisque $f(x)$ est constamment égale au lagrangien $l(x, u)$, que $x_{h}$ croît et $v_{h}=\partial l(x, u) / \partial x_{h}$ reste négative ou nulle au cours de ce passage, tandis que les autres variables $x_{j}$ restent nulles (hors de la base) ou sont associées à des $v_{j}=\partial l(x, u) / \partial x_{j}$ qui restent nulles, et qu'enfin $\nabla_{u}^{\prime} l(x, u)=A x-b$ reste nul.

Si la variable sortante est $v_{h}$, complémentaire de la variable entrante $x_{h}$ (le pivot est alors négatif), le nouveau programme de base est standard comme le précédent. (On dira qu'il s'agit d'un passage $P_{11}$.)

Si la variable sortante est $x_{k}$ (le pivot est alors positif), le nouveau programme de base est non standard : le couple $\left(v_{h}, x_{h}\right)$ est dans la base, avec $v_{h}<0$, tandis que le couple $\left(v_{k}, x_{k}\right)$ est hors de la base. (On dira qu'il s'agit d'un passage $P_{12}$.)

2) A partir d'Un PROGRamme de BASE NON STANDARD

- on fait entrer dans la base en croissant la variable $v_{k}$ figurant dans le couple $\left(v_{k}, x_{k}\right)$ de variables complémentaires hors base;

- on fait sortir de la base celle des variables de base $x_{j}$ et de la variable $v_{h}(<0)$, figurant dans le couple $\left(v_{h}, x_{h}\right)$ de variables complémentaires de base, qui s'annule la première quand $v_{k}$ croît à partir de 0 (on montrera au paragraphe 3.3 qu'alors $v_{h}$ croît et $x_{h}$ ne dícroît pas). En cas d'ambiguité entre $v_{h}$ et une variable $x_{j}$, on choisit $v_{h}$ comme variable sortante.

Ainsi est-on assuré que, dans le passage au programme de base adjacent,

- toutes les variables $x_{j}$ restent positives ou nulles (sans pouvoir croître indéfiniment puisque $v_{h}$ ne peut croître au-delà de 0 );

- la fonction $f(x)$ à minimiser décroît ou reste constante, puisque $f(x)$ est constamment égale au lagrangien $l(x, u)$, que $x_{h}$ ne décroît pas (comme on le montrera au paragraphe 3.3) et $v_{h}=\partial l(x, u) / \partial x_{h}$ reste négative ou nulle au cours de ce passage, tandis que les autres variables $x_{j}$ restent nulles ou sont associées à des $v_{j}=\partial l(x, u) / \partial x_{j}$ qui restent nulles, et qu'enfin $\nabla_{u}^{\prime} l(x, u)=A x-b$ reste nul.

Si la variable sortante est $v_{h}$ (le pivot est alors négatif), le nouveau programme de base est standard. (On dira qu'il s'agit d'un passage $P_{21}$.)

Si la variable sortante est $x_{l}$ (le pivot est alors positif), $l \neq h$ puisque $x_{h}$ ne décroît pas au cours du passage, et le nouveau programme de base est non standard comme le précédent : le couple $\left(v_{h}, x_{h}\right)$ reste dans la base, avec $v_{h}<0$, tandis que le couple $\left(v_{l}, x_{l}\right)$ est hors de la base. (On dira qu'il s'agit d'un passage $P_{22}$.)

R.A.I.R.O. Recherche opérationnelle/Operations Research 
On montrera de plus au paragraphe 3.3 que, si $x_{h}$ reste constante quand $v_{k}$ croît à partir de 0 , il en est de même pour toutes les variables $x_{j}$, de sorte que la variable sortante est $v_{h}$, et le nouveau programme de base est standard. Ce cas est le seul où la fonction $f(x)$ à minimiser reste constante (si l'on excepte les cas de dégénérescence), et il s'agit alors d'un passage $P_{21}$ de programme non standard à programme standard.

\section{3) Convergence du processus}

Il résulte de l'étude précédente qu'en l'absence de dégénérescence, les passages de programme de base à programme de base adjacent, selon l'algorithme de Gauss, tels qu'ils sont organisés dans la $2^{\mathrm{e}}$ phase de la méthode de Dantzig, donnent une suite de programmes réalisables du problème posé, la fonction $f(x)$ à minimiser décroissant strictement à chaque passage, sauf éventuellement dans certains passages $P_{21}$ isolés où elle reste constante.

Il est donc impossible de passer plusieurs fois par un même programme de base, et cela reste vrai même dans les cas de dégénérescence si l'éventualité d'un cyclage est écartée par l'un des procédés connus pour la méthode du Simplexe (cette réserve est d'ailleurs d'une faible importance pratique).

Comme les programmes de base sont en nombre fini, le processus s'achèvera en un nombre fini de passages,

- soit par la détermination des solutions extrêmes du problème posé (et de ses conditions de Kuhn et Tucker), correspondant aux programmes de base standards où $x_{j} \geqq 0, v_{j} \geqq 0, v_{j} x_{j}=0, \forall j$;

- soit par la mise en évidence de programmes réalisables du problème posé, dans lesquels $f(x)$ prend des valeurs arbitrairement petites, de sorte que $f(x)$ n'a pas de minimum fini dans ce problème.

Dans ce second cas, le processus conduit à un programme standard non optimal, à partir duquel il est possible de faire croître indéfiniment une variable hors base $x_{h}$, associée à une variable de base $v_{h}$ qui reste constante et négative, tandis que les autres variables $x_{j}$ restent positives ou nulles (on ne trouve alors aucun pivot).

Dans le cas où $x^{\prime} C x>0, \forall x \neq 0$, le problème posé admet une solution unique (cf. $\S 1.1$ et $\S 3.3$ ).

\subsection{Justification théorique de la méthode de Dantzig}

Soient $\delta x, \delta u, \delta v$ les vecteurs représentant les variations de $x, u, v$ entre $(n, 1)(m, 1)(n, 1)$

deux programmes qui vérifient les $(n+m)$ égalités linéaires figurant dans les conditions de Kuhn et Tucker du problème posé (cf. § 3.1). 
Alors

d'où

$$
\left\{\begin{array}{l}
C \delta x+A^{\prime} \delta u=\delta v \\
A \delta x=0
\end{array}\right.
$$

$$
\delta v^{\prime} . \delta x=\delta x^{\prime} . C . \delta x \geqq 0
$$

puisque la forme quadratique $x^{\prime} C x$ est semi-définie positive.

1) Dans chacun des passages de programme de base à programme de base adjacent, tels qu'ils sont organisés dans la $2^{\mathrm{e}}$ phase de la méthode de Dantzig,

$$
\delta v^{\prime} . \delta x=\delta v_{h} . \delta x_{h} \quad \text { (avec les notations du paragraphe 3.2), }
$$

car un facteur est nul dans chacun des autres termes $\delta v_{j} . \delta x_{j}(j \neq h)$ du produit scalaire $\delta v^{\prime} . \delta x$.

Ainsi $\delta v_{h} \cdot \delta x_{h} \geqq 0$, ce qui montre que, si $x_{h}$ croît, $v_{h}$ ne décroît pas.

2) Considérons un passage $P_{12}$ de programme standard à programme non standard, au cours duquel $x_{h}$ entre dans la base et $x_{k}$ sort de la base, et le passage $\left(P_{21}\right.$ ou $\left.P_{22}\right)$ qui suit $P_{12}$ et au cours duquel $v_{k}$ entre dans la base en croissant.

Compte tenu du fait que, pour toute base, les valeurs des variables de base sont déterminées sans ambiguïté par celles des variables hors base, si les variables qui se trouvent hors de la base au début du passage $P_{12}$ restent nulles à l'exception de $x_{h}$ et $v_{k}$ qui subissent des variations arbitraires $\delta x_{h}$ et $\delta v_{k}$, les variables $v_{h}$ et $x_{k}$ subissent des variations $\delta v_{h}$ et $\delta x_{k}$ déterminées en fonction linéaire de $\delta x_{h}$ et $\delta v_{k}$ :

$$
\begin{aligned}
& \delta v_{h}=\theta \delta v_{k}+\lambda \delta x_{h} \\
& \delta x_{k}=\mu \delta v_{k}+v \delta x_{h}
\end{aligned}
$$

$\theta, \lambda, \mu, v$ étant des coefficients constants, avec $v<0$ puisque, dans $P_{12}$, $\delta v_{k}=0, \delta x_{h}>0, \delta x_{k}<0$.

De plus, $\delta v^{\prime} . \delta x=\delta v_{h} . \delta x_{h}+\delta v_{k} . \delta x_{k}=\lambda\left(\delta x_{h}\right)^{2}+(\theta+v) \delta x_{h} \cdot \delta v_{k}+\mu\left(\delta v_{k}\right)^{2} \geqq 0$ quels que soient $\delta x_{h}$ et $\delta v_{k}$, ce qui implique $\lambda \geqq 0, \mu \geqq 0,4 \lambda \mu \geqq(\theta+v)^{2}$.

Ainsi, au cours $d u$ passage $P_{12}$, où $\delta v_{k}=0$ et $\delta x_{h}>0$,

$$
\delta v_{h}=\lambda \delta x_{h} \geqq 0 \quad \text { et } \quad \delta x_{k}=v \delta x_{h}<0,
$$

et, au cours du passage qui suit $P_{12}$, où $\delta v_{k}>0$ et $\delta x_{k}=0$,

$$
\delta v_{h}=\left(\theta-\frac{\lambda \mu}{v}\right) \delta v_{k} \quad \text { et } \quad \delta x_{h}=-\frac{\mu}{v} \delta v_{k} \geqq 0 .
$$

R.A.I.R.O. Recherche opérationnelle/Operations Research 
De plus, d'après les signes de $\lambda, \mu, v$, et la condition $4 \lambda \mu \geqq(\theta+v)^{2}$,

$$
\theta-\frac{\lambda \mu}{v} \geqq \theta-\frac{(\theta+v)^{2}}{4 v}=-\frac{(\theta-v)^{2}}{4 v} \geqq 0 .
$$

Si $(\theta-(\lambda \mu / v))$ était $n u l$, il en résulterait

$$
\theta=v<0 \quad \text { et } \quad \lambda \mu=v^{2}>0,
$$

d'où, au cours du passage qui suit $P_{12}, \delta v_{h}=0, \delta x_{h}>0$, et $\delta v^{\prime} . \delta x=0$, ce qui implique $\delta x^{\prime} . C . \delta x=0$ et $C \delta x=0(c f . \S 1.1)$, de sorte que $\left\{\begin{array}{c}A^{\prime} \delta u=\delta v, \\ A \delta x=0 .\end{array}\right.$

En particulier, les relations $C \delta x=0$ et $A \delta x=0$, jointes à la nullité de $\delta x_{j}$ au cours de ce passage pour toute variable $x_{j}$ hors base, déterminent sans ambiguité $\delta x=0$, ce qui est contradictoire avec $\delta x_{h}>0$.

Donc $\theta-(\lambda \mu / v)>0$ et $\delta v_{h}>0$ au cours du passage qui suit $P_{12}$.

Il est ainsi établi que (sauf en cas de dégénérescence) :

$$
\begin{array}{|llll}
\delta x_{h}>0 & \text { et } & \delta v_{h} \geqq 0 & \text { au cours du passage } P_{12} \\
\delta x_{h} \geqq 0 & \text { et } & \delta v_{h}>0 & \text { au cours du passage qui suit } P_{12}
\end{array}
$$

Si $\delta v_{h}=0$ au cours $d u$ passage $P_{12}$, c'est-à-dire si $\lambda=0, \delta v^{\prime} . \delta x=0$, ce qui implique $\delta x^{\prime} . C . \delta x=0$ et $C \delta x=0$, de sorte que $\left\{\begin{array}{c}A^{\prime} \delta u=\delta v \\ A \delta x=0\end{array}\right\}$ au cours de $P_{12}$. En particulier, la relation $A^{\prime} \delta u=\delta v$, jointe à la nullité de $\delta v_{j}$ au cours de $\mathrm{P}_{12}$ pour toute variable $v_{j}$ hors base, détermine sans ambiguïté $\delta u=0$ et $\delta v=0$. Ainsi, si $\delta v_{h}=0$, tous les $u_{i}$ et tous les $v_{j}$ restent constants au cours du passage $P_{12}$.

Ce cas est exclu si $\left\{\begin{array}{l}C \delta x=0 \\ A \delta x=0\end{array}\right\} \Rightarrow[\delta x=0]$ (ce qui est contradictoire avec $\delta x_{h}>0$ ), et en particulier si la forme quadratique $x^{\prime} C x$ est définie positive. Alors $\delta v_{h}>0$ au cours de $P_{12}$, ce qui exclut que la variable $x_{h}$ puisse croître indéfiniment sans que $v_{h}$ devienne positif.

Si $\delta x_{h}=0$ au cours $d u$ passage qui suit $P_{12}$, c'est-à-dire si $\mu=0, \delta v^{\prime} . \delta x=0$, ce qui implique $\delta x^{\prime} . C . \delta x=0$ et $C \delta x=0$, de sorte que $\left\{\begin{array}{l}A^{\prime} \delta u=\delta v \\ A \delta x=0\end{array}\right\}$ au cours de ce passage. En particulier, les relations $C \delta x=0$ et $A \delta x=0$, jointes à la nullité de $\delta x_{j}$ vol. $13, \mathrm{n}^{\circ} 2$, mai 1979 
au cours de ce passage pour toute variable $x_{j}$ hors base, déterminent sans ambiguïté $\delta x=0$. Ainsi, si $\delta x_{h}=0$, tous les $x_{j}$ restent constants au cours $d u$ passage qui suit $P_{12}$, de sorte qu'il s'agit d'un passage $P_{21}$ conduisant à un programme standard : $v_{h}$ croît à partir d'une valeur négative et sort de la base en s’annulant.

3) Si un passage $P_{12}$, au cours duquel $x_{h}$ entre dans la base et $x_{k}$ sort de la base, est suivi d'un passage $P_{22}$, au cours duquel $v_{k}$ entre dans la base et $x_{l}$ sort de la base, $\delta x_{h}>0, \delta v_{h}>0$ et $\delta x_{l}<0$ au cours de $P_{22}$ tandis que $\delta v_{k}>0$, et, au cours du passage $\left(P_{21}\right.$ ou $\left.P_{22}\right)$ qui suit $P_{22}, v_{l}$ entre dans la base en croissant.

Si les variables qui se trouvent hors de la base au début du passage $P_{22}$ restent nulles à l'exception de $v_{k}$ et $v_{l}$ qui subissent des variations arbitraires $\delta v_{k}$ et $\delta v_{l}$, les variables $x_{h}, v_{h}$ et $x_{l}$ subissent des variations $\delta x_{h}, \delta v_{h}$ et $\delta x_{l}$ déterminées en fonction linéaire de $\delta v_{k}$ et $\delta v_{l}$. Mais, puisque $\delta x_{h}>0$ quand $\delta v_{k}>0$ et $\delta v_{l}=0$ (au cours de $P_{22}$ ), on peut choisir comme variables indépendantes $\partial x_{h}$ et $\partial v_{l}$ au lieu de $\delta v_{k}$ et $\delta v_{l}$, et exprimer $\delta v_{h}$ et $\delta x_{l}$ en fonction linéaire de $\delta x_{h}$ et $\delta v_{l}$ :

$$
\begin{aligned}
& \delta v_{h}=\theta^{\prime} \delta v_{l}+\lambda^{\prime} \delta x_{h} \\
& \delta x_{l}=\mu^{\prime} \delta v_{l}+v^{\prime} \delta x_{h}
\end{aligned}
$$

$\theta^{\prime}, \lambda^{\prime}, \mu^{\prime}, v^{\prime}$ étant des coefficients constants, avec $\left\{\begin{array}{l}\lambda^{\prime}>0 \\ v^{\prime}<0\end{array}\right\}$ puisque, dans $P_{22}, \delta v_{l}=0, \delta x_{h}>0,\left\{\begin{array}{l}\delta v_{h}>0, \\ \delta x_{l}<0 .\end{array}\right.$

De plus, puisque $\delta x_{k}=0, \quad \delta v^{\prime} . \delta x=\delta v_{h} . \delta x_{h}+\delta v_{l} . \delta x_{l} \geqq 0, \forall\left(\delta x_{h}, \delta v_{l}\right)$.

On peut donc reprendre ici, pour $P_{22}$ et le passage qui le suit, l'étude faite en 2) pour $P_{12}$ et le passage qui le suit, en remplaçant $\theta, \lambda, \mu, v$ par $\theta^{\prime}, \lambda^{\prime}, \mu^{\prime}, v^{\prime}$ et $x_{k}, v_{k}$ par $x_{l}, v_{l}$.

Il est ainsi établi que (sauf en cas de dégénérescence) :

$$
\begin{array}{|llll|}
\hline \partial x_{h}>0 & \text { et } & \delta v_{h}>0 & \text { au cours du passage } P_{22}, \\
\delta x_{h} \geqq 0 & \text { et } & \delta v_{h}>0 & \text { au cours du passage qui suit } P_{22}
\end{array}
$$

et que $\delta x_{h}$ ne peut être nul au cours de ce dernier passage que si c'est un passage $P_{21}$.

Par récurrence, ces résultats restent valables pour toute suite de passages $P_{22}$ succédant à $P_{12}$. 


\section{4. Note sur le calcul de $f(x)$ dans la méthode de Dantzig}

D'après le calcul fait au paragraphe 1.3 et compte tenu des variables artificielles, coordonnées de $w$, éventuellement introduites dans les conditions de Kuhn et Tucker (cf. $\S 3.2)$,

$$
2 f(x)=p^{\prime} x-u^{\prime} b+v^{\prime} x+u^{\prime} w
$$

A la fin de la $1^{\text {re }}$ phase du processus, $w=0$, et dans tout programme de base $(m, 1)$ standard, en particulier dans tout programme extrême optimum, $v^{\prime} x=0$.

Il est donc intéressant d'ajouter une ligne dans les tableaux de calcul, pour calculer, au cours du processus, l'expression $p^{\prime} x-u^{\prime} b$, linéaire en $\underset{(n, 1)}{x}$ et $\underset{(1, m)}{u^{\prime}}$, qui donnera, à la fin du processus, la valeur optimale de $2 f(x)$ (quand elle existe).

Cette ligne supplémentaire permet de suivre l'évolution de la fonction économique au cours de la $2^{\mathrm{e}}$ phase du processus, $2 f(x)$ étant égal à $\left(p^{\prime} x-u^{\prime} b\right)$ dans chaque programme standard, et à $\left(p^{\prime} x-u^{\prime} b\right)$ augmenté du seul terme $v_{j} x_{j}$ non nul dans chaque programme non standard.

\section{EXEMPLE}

\subsection{Problème}

$\lambda$ étant un paramètre réel positif ou nul.

$$
\text { Minimiser } f(\lambda, x)=-\lambda\left(5 x_{1}+2 x_{2}\right)+\frac{1}{18}\left(x_{1}-2 x_{2}\right)^{2},
$$

sous les contraintes :

$$
\begin{aligned}
& \left\{\begin{array}{c}
x_{1}+x_{2} \leqq 12 \\
4 x_{1}+x_{2} \leqq 24 \\
x_{1}, x_{2} \geqq 0
\end{array}\right\}, \quad \text { ou } \quad\left\{\begin{array}{cc}
x_{1}+x_{2}+x_{3} & =12 \\
4 x_{1}+x_{2}+ & x_{4}=24 \\
x_{1}, x_{2}, x_{3}, & x_{4} \geqq 0
\end{array}\right\} \\
& \text { (après introduction } \\
& \text { des variables d'écart } x_{3}, x_{4} \text { ). }
\end{aligned}
$$

Le lagrangien (formé sans tenir compte des conditions de signe $x_{j} \geqq 0$ ) s "écrit :

$$
\begin{aligned}
l(x, u)=-\lambda\left(5 x_{1}+2 x_{2}\right)+\frac{1}{18}( & \left.x_{1}-2 x_{2}\right)^{2} \\
& +u_{3}\left(x_{1}+x_{2}+x_{3}-12\right)+u_{4}\left(4 x_{1}+x_{2}+x_{4}-24\right),
\end{aligned}
$$

vol. $13, \mathrm{n}^{\circ} 2$, mai 1979 
et l'on en déduit les conditions de Kuhn et Tucker (nécessaires et suffisantes) :

ou

$$
\left\{\begin{array}{cr}
-5 \lambda+\frac{x_{1}}{9}-\frac{2 x_{2}}{9}+u_{3}+4 u_{4}=v_{1}, \\
-2 \lambda-\frac{2 x_{1}}{9}+\frac{4 x_{2}}{9}+u_{3}+u_{4}=v_{2}, \\
u_{3} \quad=v_{3}, \\
u_{4}=v_{4}, \\
x_{1}+x_{2}+x_{3} & =12, \\
4 x_{1}+x_{2}+x_{4} & =24, \\
x_{j} \geqq 0, \quad v_{j} x_{j}=0, & \forall j,
\end{array}\right\}
$$

$$
\begin{array}{|c|}
\frac{x_{1}}{9}-\frac{2 x_{2}}{9}+v_{3}+4 v_{4}-v_{1} \quad=5 \lambda, \\
-\frac{2 x_{1}}{9}+\frac{4 x_{2}}{9}+v_{3}+v_{4} \quad-v_{2}=2 \lambda, \\
x_{1}+x_{2}+x_{3}=12, \\
4 x_{1}+x_{2} \quad x_{4}=24, \\
x_{j} \geqq 0, \quad v_{j} x_{j}=0, \quad \forall j \in\{1,2,3,4\}, \\
\hline
\end{array}
$$

après élimination (immédiate) des deux multiplicateurs $u_{3}$ et $u_{4}$, de signes quelconques (a priori).

Compte tenu des quatre égalités linéaires précédentes $(c f . \S 1.3)$,

$$
2 f(\lambda, x)=-\lambda\left(5 x_{1}+2 x_{2}\right)-12 v_{3}-24 v_{4}+\sum_{j=1}^{4} v_{j} x_{j}
$$

le dernier terme (non linéaire) étant nul quand les relations d'exclusion, $v_{j} x_{j}=0, \forall j$, sont vérifiées.

\subsection{Application de la méthode de Wolfe complétée}

Un programme de base réalisable daris le problème initial est en évidence :

$$
x_{1}=0, \quad x_{2}=0, \quad x_{3}=12, \quad x_{4}=24,
$$

ce qui dispense de la $1^{\text {re }}$ phase du processus.

R.A.I.R.O. Recherche opérationnelle/Operations Research 


\subsubsection{Forme COURTE DU PROCESSUS POUR $\lambda=1$}

On ajoute les variables artificielles respectives $z_{1}$ et $z_{2}$ aux premiers membres de la première et de la seconde équation de Kuhn et Tucker, et les termes $-z_{1} x_{1}-z_{2} x_{2}$ à la dernière expression de $2 f(\lambda, x)(c f . \S 2.5)$.

Les tableaux de calcul sont les suivants :

\begin{tabular}{l|rrrrrr|r} 
& $x_{1}$ & $x_{2}$ & $v_{3}$ & $v_{4}$ & $v_{1}$ & $v_{2}$ & \\
\hline$z_{1}+z_{2}$ & $-\frac{1}{9}$ & $\frac{2}{9}$ & 2 & 5 & -1 & -1 & 7 \\
\hline$z_{1}$ & $\frac{1}{9}$ & $-\frac{2}{9}$ & 1 & 4 & -1 & 0 & 5 \\
$z_{2}$ & $-\frac{2}{9}$ & $\frac{4}{9}$ & 1 & 1 & 0 & -1 & 2 \\
$x_{3}$ & 1 & 1 & 0 & 0 & 0 & 0 & 12 \\
$x_{4}$ & 4 & 1 & 0 & 0 & 0 & 0 & 24 \\
\hline $2 f$ & 5 & 2 & 12 & 24 & 0 & 0 & 0
\end{tabular}

\begin{tabular}{l|c|rrrrr|r} 
& $x_{1}$ & $z_{2}$ & $v_{3}$ & $v_{4}$ & $v_{1}$ & $v_{2}$ & \\
\hline$z_{1}+z_{2}$ & 0 & $-\frac{1}{2}$ & $\frac{3}{2}$ & $\frac{9}{2}$ & -1 & $-\frac{1}{2}$ & 6 \\
\hline$z_{1}$ & 0 & $\frac{1}{2}$ & $\frac{3}{2}$ & $\frac{9}{2}$ & -1 & $-\frac{1}{2}$ & 6 \\
$x_{2}$ & $-\frac{1}{2}$ & $\frac{9}{4}$ & $\frac{9}{4}$ & $\frac{9}{4}$ & 0 & $-\frac{9}{4}$ & $\frac{9}{2}$ \\
$x_{3}$ & $\frac{3}{2}$ & $-\frac{9}{4}$ & $-\frac{9}{4}$ & $-\frac{9}{4}$ & 0 & $\frac{9}{4}$ & $\frac{15}{2}$ \\
$x_{4}$ & $\frac{9}{2}$ & $-\frac{9}{4}$ & $-\frac{9}{4}$ & $-\frac{9}{4}$ & 0 & $\frac{9}{4}$ & $\frac{39}{2}$ \\
\hline $2 f$ & 6 & $-\frac{9}{2}$ & $\frac{15}{2}$ & $\frac{39}{2}$ & 0 & $\frac{9}{2}$ & -9
\end{tabular}

\begin{tabular}{l|r|rrrrr|r} 
& $x_{4}$ & $z_{2}$ & $v_{3}$ & $v_{4}$ & $v_{1}$ & $v_{2}$ & \\
\hline$z_{1}+z_{2}$ & 0 & $-\frac{1}{2}$ & $\frac{3}{2}$ & $\frac{9}{2}$ & -1 & $-\frac{1}{2}$ & 6 \\
\hline$z_{1}$ & 0 & $\frac{1}{2}$ & $\frac{3}{2}$ & $\frac{9}{2}$ & -1 & $-\frac{1}{2}$ & 6 \\
$x_{2}$ & $\frac{1}{9}$ & 2 & 2 & 2 & 0 & -2 & $\frac{20}{3}$ \\
$x_{3}$ & $-\frac{1}{3}$ & $-\frac{3}{2}$ & $-\frac{3}{2}$ & $-\frac{3}{2}$ & 0 & $\frac{3}{2}$ & 1 \\
$x_{1}$ & $\frac{2}{9}$ & $\frac{1}{2}$ & $-\frac{1}{2}$ & $-\frac{1}{2}$ & 0 & $\frac{1}{2}$ & $\frac{13}{3}$ \\
\hline $2 f$ & $-\frac{4}{3}$ & $\frac{1}{2}$ & $\frac{21}{2}$ & $\frac{45}{2}$ & 0 & $\frac{3}{2}$ & -35 \\
& & & & & & & -26
\end{tabular}

\begin{tabular}{|c|c|c|c|c|c|c|c|}
\hline & $x_{+}$ & $z_{3}$ & $r_{3}$ & $z_{1}$ & $v_{1}$ & $v_{2}$ & \\
\hline$z_{1}+z_{2}$ & 0 & -1 & 0 & -1 & 0 & 0 & 0 \\
\hline \multirow{2}{*}{$v_{4}$} & \multirow{2}{*}{0} & & 1 & 2 & 2 & 1 & 4 \\
\hline & & & 3 & 9 & 9 & $\overline{9}$ & 3 \\
\hline \multirow{2}{*}{$x_{2}$} & 1 & 16 & 4 & 4 & 4 & 16 & 4 \\
\hline & 9 & 9 & 3 & 9 & $\overline{9}$ & $\overline{9}$ & 4 \\
\hline \multirow{2}{*}{$x_{3}$} & 1 & 4 & -1 & 1 & -1 & 4 & 3 \\
\hline & 3 & 3 & & 13 & 3 & 3 & \\
\hline \multirow{2}{*}{$x_{1}$} & 2 & 4 & 1 & X. & $-\frac{1}{2}$ & 4 & 5 \\
\hline & 9 & 9 & 3 & .8 & $\overline{9}$ & 9 & \\
\hline $2 f$ & & 4 & 3 & -5 & 5 & 4 & -65 \\
\hline
\end{tabular}

La solution unique du problème initial est atteinte au $4^{\mathrm{e}}$ tableau (pour $\left.\lambda=1\right)$ :

$$
\bar{x}_{1}=5, \quad \bar{x}_{2}=4, \quad \text { et } \quad f(1, \bar{x})=-65 / 2 \text {. }
$$

vol. $13, \mathrm{n}^{\circ} 2$, mai 1979 
Remarques. - L'élément encerclé dans chaque tableau autre que le dernier est le pivot.

Dès qu'une variable artificielle $z_{1}$ ou $z_{2}$ sort de la base, elle pourrait être supprimée.

Quand $\left(-z_{1} x_{1}-z_{2} x_{2}\right) \neq 0$ ( $3^{\mathrm{e}}$ tableau), sa valeur est inscrite sous la valeur de la partie linéaire de $2 f$ calculée par l'algorithme du Simplexe.

Le second tableau de calcul conduit à un blocage apparent, puisque $x_{1}$ ou $v_{1}$ peuvent seuls entrer dans la base et que leur croissance à partir de 0 ne peut faire décroître $\left(z_{1}+z_{2}\right)$. Mais ce blocage n'est qu'apparent, car l'entrée de $x_{1}$ dans la base permet de passer à un tableau non bloqué (cf. § 2.4.2).

Si l'on conservait les seules contraintes de signe dans le problème initial, les deux premiers tableaux de calcul resteraient valables si l'on y supprimait les lignes $x_{3}, x_{4}$ et les colonnes $v_{3}, v_{4}$. Le second tableau permettrait alors une croissance indéfinie de $x_{1}$, au cours de laquelle $f(1, x)$ décroîtrait indéfiniment, avec $2 x_{2}-x_{1}=9$.

\subsubsection{FORME LONGUE DU PROCESSUS}

Le programme de base réalisable qui est en évidence dans le problème initial en est une solution extrême pour $\lambda=0$, ce qui dispense de la $1^{\text {re }}$ et de la $2^{\mathrm{e}}$ phases du processus. Il ne reste qu'à essayer de faire croître $\lambda$ à partir de 0 .

Les tableaux de calcul se trouvent à la page 181 .

Le problème paramétrique initial est résolu par les $4^{\mathrm{e}}$ et $5^{\mathrm{e}}$ tableaux (cf. $\$ 2.4 .3)$ :

- pour $\lambda=0$,

$$
\bar{x}_{1}=\frac{16}{3} \alpha, \quad \bar{x}_{2}=\frac{8}{3} \alpha, \quad \forall \alpha \in[0,1], \quad \text { et } \quad f(0, \bar{x})=0 ;
$$

- pour $\lambda \in[0,4]$,

$$
\left\{\begin{array}{l}
\quad \bar{x}_{1}=\frac{16}{3} \frac{4-\lambda}{4}+4 \frac{\lambda}{4}=\frac{16-\lambda}{3}, \quad \bar{x}_{2}=\frac{8}{3} \frac{4-\lambda}{4}+8 \frac{\lambda}{4}=\frac{8+4 \lambda}{3}, \\
\text { et } \quad f(\lambda, \bar{x})=-16 \lambda \frac{4-\lambda}{4}-(64+18 \lambda) \frac{\lambda}{4}=-32 \lambda-\frac{\lambda^{2}}{2} ;
\end{array}\right.
$$

(On pourrait aussi calculer $\bar{x}_{3}, \bar{x}_{4}, \bar{v}_{1}, \bar{v}_{2}, \bar{v}_{3}, \bar{v}_{4}$ en fonction de $\lambda \geqq 0$.)

Remarques. - Les trois premiers tableaux correspondent au programme de départ, qui est dégénéré, avec $\lambda=0$, mais il n'y a pas de cyclage. (On aurait pu commencer par échanger $\lambda$ et $v_{2}$, au lieu de $\lambda$ et $v_{1}$.)

R.A.I.R.O. Recherche opérationnelle/Operations Research 
PROGRAMMATION QUADRATIQUE CONVEXE

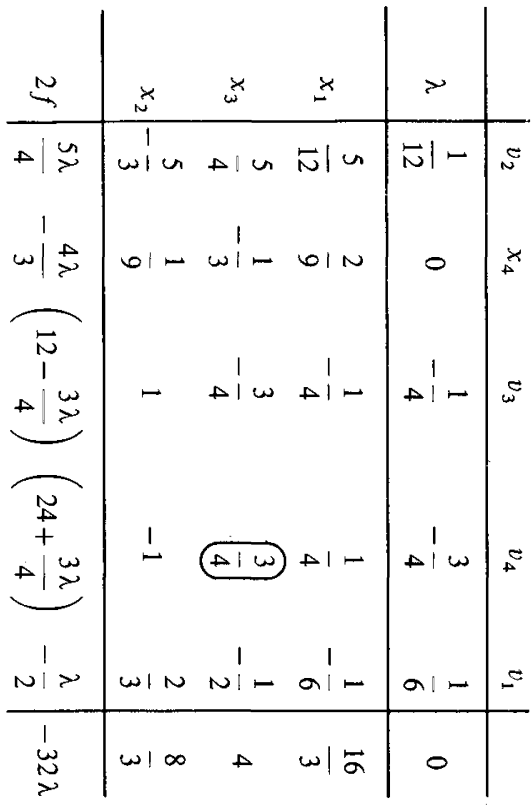

\begin{tabular}{|c|c|c|c|c|c|}
\hline$\underbrace{N}$ & $\stackrel{x}{x}$ & $\omega$ & $\stackrel{\sim}{\sim}$ & 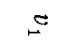 & \\
\hline$\ddot{\tau}$ & $A$ & - & $0 ! N$ & - & $\stackrel{x}{ }$ \\
\hline$\stackrel{\sim}{\nearrow}$ & - & $\leftarrow$ & o' & OIN & $\stackrel{\sim}{\sim}$ \\
\hline$\vec{N}$ & 0 & 0 & I & I & E \\
\hline$\tilde{A}$ & 0 & 0 & $\stackrel{1}{\sim}$ & $\stackrel{1}{b}$ & 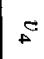 \\
\hline 0 & 0 & 0 & N & (n) & $>$ \\
\hline & $\tilde{\Phi}$ & $\tilde{N}$ & 0 & & \\
\hline
\end{tabular}

\begin{tabular}{|c|c|c|c|c|c|}
\hline$\underbrace{N}$ & $\stackrel{n}{x}$ & 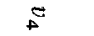 & $\check{x}$ & $>$ & \\
\hline $\begin{array}{l}1 \\
t\end{array}$ & 0 & & 0 & $\omega \mid \rightarrow$ & N \\
\hline$\omega||_{1}^{\omega}$ & wir & هit & w| - & wI & 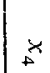 \\
\hline$\tilde{\alpha}$ & 0 & $\stackrel{I}{\leftarrow}$ & 0 & 1 & 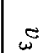 \\
\hline$\underset{N}{\sim}$ & $\omega \mid \triangle$ & $\omega \mid A$ & wI & - & $\omega_{\omega}^{x}$ \\
\hline $\bar{a}$ & 0 & win & 0 & w! & $\approx$ \\
\hline 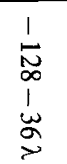 & $\infty$ & $\omega / \sigma$ & $A$ & $\rightarrow$ & \\
\hline$\underset{\omega}{\stackrel{1}{\omega}}$ & $\stackrel{t}{0}$ & $\underset{\omega}{+}$ & $\stackrel{+}{0}$ & $\underset{\omega}{t}$ & \\
\hline
\end{tabular}

\begin{tabular}{|c|c|c|c|c|c|}
\hline$\stackrel{\sim}{\sim}$ & $\stackrel{x}{\star}$ & w. & 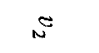 & $>$ & \\
\hline$\underset{c}{c}$ & A & $\mapsto$ & $\vec{E} \mid \oplus$ & 点| & $\stackrel{x}{*}$ \\
\hline$\stackrel{N}{>}$ & - & 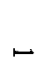 & $\vec{n} \mid \infty$ & $\stackrel{n}{n} N$ & $\stackrel{x}{N}$ \\
\hline $\bar{N}$ & 0 & 0 & n'w & u't & $\omega$ \\
\hline$\tilde{A}$ & 0 & 0 & nा w & uis & 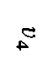 \\
\hline 0 & 0 & 0 & un & un 1 - & $\approx$ \\
\hline & 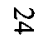 & $\bar{N}$ & 0 & 0 & \\
\hline
\end{tabular}

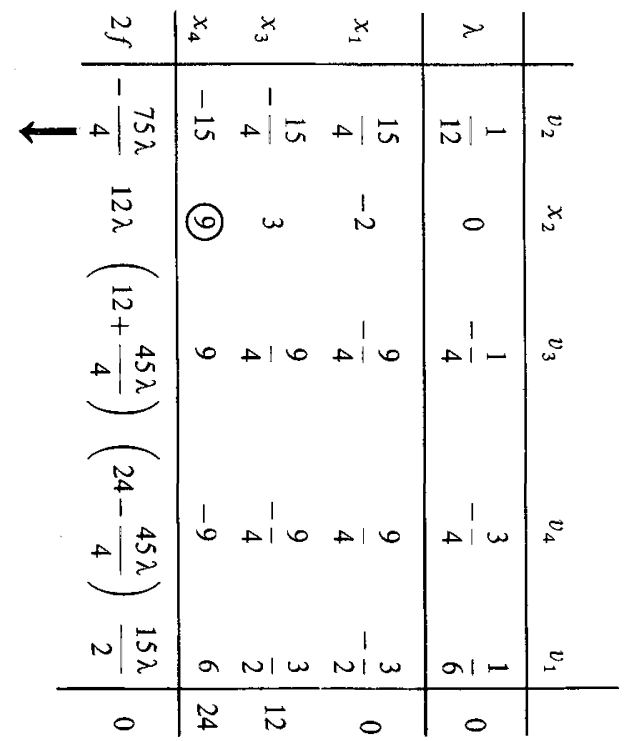

vol. $13, \mathrm{n}^{\circ} 2$, mai 1979 
Le troisième tableau de calcul conduit à un blocage apparent, puisque $v_{2}$ ou $x_{2}$ peuvent seuls entrer dans la base et que leur croissance à partir de 0 ne peut faire croître $\lambda$. Mais ce blocage n'est qu'apparent, car l'entrée de $x_{2}$ dans la base permet de passer à un tableau non bloqué ( $c f . \S 2.4 .3$ ).

Dans le dernier tableau, la variable hors base $v_{3}$, qui peut croître indéfiniment à partir de 0 , est marquée d'une flèche, et la dernière colonne donne les accroissements qui en résultent pour les variables de base et pour $2 f$.

Si l'on conservait les seules contraintes de signe dans le problème paramétrique initial, les trois premiers tableaux de calcul resteraient valables si l'on y supprimait les lignes $x_{3}, x_{4}$ et les colonnes $v_{3}, v_{4}$. Le troisième tableau permettrait alors une croissance indéfinie de $x_{2}, \lambda$ restant nul, avec $x_{1}-2 x_{2}=0$, et, pour tout $\lambda>0, f(\lambda, x)$ décroîtrait alors indéfiniment.

\subsection{Application de la méthode de Dantzig pour $\lambda=1$}

Le fait qu'un programme de base réalisable soit en évidence dans le problème posé dispense de la $1^{\mathrm{re}}$ phase du processus.

Les tableaux de calcul se trouvent à la page 183.

La solution unique du problème posé est atteinte au $4^{\mathrm{e}}$ tableau :

$$
\bar{x}_{1}=5, \quad \bar{x}_{2}=4, \quad \text { et } \quad f(1, \bar{x})=-65 / 2 .
$$

Remarques. - Quand $\sum_{j=1}^{4} v_{j} x_{j} \neq 0\left(3^{\mathrm{e}}\right.$ tableau $)$, la valeur du seul terme $v_{j} x_{j}$ non nul dans le programme non standard correspondant est inscrite sous la valeur de la partie linéaire de $2 f$ calculée par l'algorithme du Simplexe.

Si l'on conservait les seules contraintes de signe dans le problème posé, les deux premiers tableaux de calcul resteraient valables si l'on y supprimait les lignes $x_{3}, x_{4}$ et les colonnes $v_{3}, v_{4}$. Le second tableau permettrait alors une croissance indéfinie de $x_{1}$, au cours de laquelle $f(1, x)$ décroîtrait indéfiniment, la variable $v_{1}$ associée à $x_{1}$ restant constante et négative, avec $2 x_{2}-x_{1}=9$.

$N . B$. : La règle pratique généralement adoptée aurait conduit à commencer par faire entrer dans la base la variable hors base $x_{1}$ associée à $v_{1}$, qui est la variable $v_{j}$ négative de plus grande valeur absolue. Mais cette règle ne s'impose pas: on peut vérifier qu'ici, son application n'abrégerait pas (et n'allongerait pas) le calcul.

En commençant par faire entrer $x_{2}$ dans la base, on obtient ici des tableaux de calcul immédiatement comparables à ceux obtenus dans l'application de la forme courte de la méthode de Wolfe (cf. § 4.2). Cette comparaison montre que la 


\begin{tabular}{l|rrrr|rr|rrrr|r} 
& $x_{1}$ & $x_{2}$ & $v_{3}$ & $v_{4}$ & & & $x_{1}$ & $v_{2}$ & $v_{3}$ & $v_{4}$ & \\
\hline$v_{1}$ & $-\frac{1}{9}$ & $\frac{2}{9}$ & -1 & -4 & -5 & $v_{1}$ & 0 & $\frac{1}{2}$ & $-\frac{3}{2}$ & $-\frac{9}{2}$ & -6 \\
$v_{2}$ & $\frac{2}{9}$ & $-\frac{4}{9}$ & -1 & -1 & -2 & $x_{2}$ & $-\frac{1}{2}$ & $-\frac{9}{4}$ & $\frac{9}{4}$ & $\frac{9}{4}$ & $\frac{9}{2}$ \\
$x_{3}$ & 1 & 1 & 0 & 0 & 12 & $x_{3}$ & $\frac{3}{2}$ & $\frac{9}{4}$ & $-\frac{9}{4}$ & $-\frac{9}{4}$ & $\frac{15}{2}$ \\
$x_{4}$ & 4 & 1 & 0 & 0 & 24 & $x_{4}$ & $\frac{9}{2}$ & $\frac{9}{4}$ & $-\frac{9}{4}$ & $-\frac{9}{4}$ & $\frac{39}{2}$ \\
\hline $2 f$ & 5 & 2 & 12 & 24 & 0 & $2 f$ & 6 & $\frac{9}{2}$ & $\frac{15}{2}$ & $\frac{39}{2}$ & -9
\end{tabular}

\begin{tabular}{|c|c|c|c|c|c|c|c|c|c|c|c|}
\hline & $x_{4}$ & $v_{2}$ & $v_{3}$ & $v_{4}$ & & & $x_{4}$ & $v_{2}$ & $v_{3 \ldots}$ & $v_{1}$ & \\
\hline \multirow[b]{2}{*}{$v_{1}$} & & 1 & 3 & 9 & & \multirow[b]{2}{*}{$v_{4}$} & \multirow{2}{*}{0} & 1 & 1 & 2 & \\
\hline & 0 & $\overline{2}$ & $-\overline{2}$ & $\left(-\frac{1}{2}\right)$ & & & & $-\overline{9}$ & $\overline{3}$ & $\overline{9}$ & $\overline{3}$ \\
\hline \multirow{2}{*}{$x_{2}$} & 1 & -2 & 2 & 2 & 20 & \multirow{2}{*}{$x_{2}$} & 1 & -16 & $\underline{4}$ & $\underline{4}$ & 4 \\
\hline & $\overline{9}$ & -2 & 2 & & $\overline{3}$ & & $\overline{9}$ & $\overline{9}$ & $\overline{3}$ & $\overline{9}$ & 4 \\
\hline \multirow{2}{*}{$x_{3}$} & $-\frac{1}{1}$ & 3 & -3 & -3 & 1 & \multirow{2}{*}{$x_{3}$} & 1 & $\underline{4}$ & -1 & 1 & 3 \\
\hline & $\overline{3}$ & 2 & $\overline{2}$ & $\overline{2}$ & 1 & & $\overline{3}$ & $\overline{3}$ & -1 & $\overline{3}$ & 3 \\
\hline \multirow[b]{2}{*}{$x_{1}$} & 2 & 1 & 1 & 1 & 13 & \multirow[b]{2}{*}{$x_{1}$} & 2 & 4 & 1 & 1 & \\
\hline & 9 & $\overline{2}$ & $\overline{2}$ & 2 & 3 & & $\overline{9}$ & $\overline{9}$ & $\overline{3}$ & $\overline{9}$ & 5 \\
\hline \multirow{2}{*}{$2 f$} & 4 & 3 & 21 & 45 & \multirow{2}{*}{$\begin{array}{l}-35 \\
-26\end{array}$} & \multirow{2}{*}{$2 f$} & 4 & \multirow{2}{*}{4} & \multirow{2}{*}{3} & \multirow{2}{*}{5} & \multirow{2}{*}{-65} \\
\hline & 3 & 2 & 2 & 2 & & & 3 & & & & \\
\hline
\end{tabular}

méthode de Dantzig conduit à des calculs plus réduits, en évitant d'introduire des variables artificielles.

(En revanche, la forme longue de la méthode de Wolfe permet de résoudre le problème paramétrique.)

\section{BIBLIOGRAPHIE}

1. E. W. Barankin et R. Dorfman, On Quadratic Programming, University of California Publications in Statistics, vol. 2, 1958, p. 285-318.

2. J. C. G. Boot, Programmation quadratique : algorithmes, anomalies, applications, North-Holland Publishing Company, 1964 et Dunod, 1968.

3. G. B. DANTZIG, Quadratic Programming: a Variant of the Wolfe-Markowitz Algorithms, Research Report 2 of the Operations Research Center of the University of California, Berkeley, 1961. 
4. G. B. Dantzig, Applications et prolongements de la programmation linéaire, Princeton University Press, 1963 et Dunod, 1966.

5. W. S. Dorn, Nonlinear Programming: a Survey, Management Science, vol. 9, 1963, p. 171-208.

6. E. L. Keller, The General Quadratic Optimization Problem, Mathematical Programming, vol. 5, 1973, p. 311-337.

7. H. P. Künzı, W. Krelle et W. Oettlı, La programmation non linéaire, Blaisdell Publishing Company, 1966 et Gauthier-Villars, 1969.

8. C. Van de Panne et A. Whinston, The Simplex and the Dual Method for Quadratic Programming, Report 6314 of the Econometric Institute of the Netherlands School of Economics, Rotterdam, 1963.

9. Ph. Wolfe, The Simplex Method for Quadratic Programming, Econometrica, vol. 27, 1959, p. 382-398. 